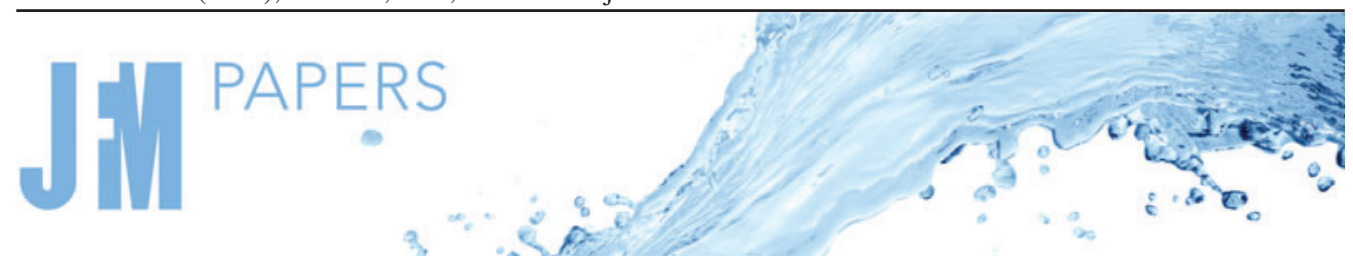

\title{
$Q$-tensor model for undulatory swimming in lyotropic liquid crystal polymers
}

\author{
Zhaowu Lin ${ }^{1,2}$, Sheng Chen ${ }^{2}$ and Tong Gao ${ }^{2,3}, \dagger$ \\ ${ }^{1}$ Department of Engineering Mechanics, Zhejiang University, Hangzhou, PR China \\ ${ }^{2}$ Department of Mechanical Engineering, Michigan State University, East Lansing, MI 48864, USA \\ ${ }^{3}$ Department of Computational Mathematics, Science and Engineering, Michigan State University, \\ East Lansing, MI 48864, USA
}

(Received 31 January 2021; revised 15 April 2021; accepted 9 June 2021)

Microorganisms may exhibit rich swimming behaviours in anisotropic fluids, such as liquid crystals, which have direction-dependent physical and rheological properties. Here we construct a two-dimensional computation model to study the undulatory swimming mechanisms of microswimmers in a solution of rigid, rodlike liquid crystal polymers. We describe the fluid phase using Doi's $Q$-tensor model, and treat the swimmer as a finite-length flexible fibre with imposed propagating travelling waves on the body curvature. The fluid-structure interactions are resolved via an immersed boundary method. Compared with the swimming dynamics in Newtonian fluids, we observe non-Newtonian behaviours that feature both enhanced and retarded swimming motions in lyotropic liquid crystal polymers. We reveal the propulsion mechanism by analysing the near-body flow fields and polymeric force distributions, together with asymptotic analysis for an idealized model of Taylor's swimming sheet.

Key words: micro-organism dynamics, liquid crystals

\section{Introduction}

Many organisms live in microfluidic environments, either biological or synthetic, where the fluid inertia is negligible. In the so-called Stokes (or creeping) flows, Purcell's scallop theorem explains that performing time-reversible motions cannot generate directional swimming or locomotion owing to kinematic reversibility (Purcell 1977). Instead, microswimmers often adopt special propulsion strategies (i.e. swimming gaits). Especially for those with slender shapes or having thin appendages (e.g. C. elegans, E. coli, B. subtilis), it is well-understood that they can perform undulation or flagellar

$\dagger$ Email address for correspondence: gaotong@egr.msu.edu

(C) The Author(s), 2021. Published by Cambridge University Press. This is an Open Access article, distributed under the terms of the Creative Commons Attribution licence (http://creativecommons.org/ licenses/by/4.0/), which permits unrestricted re-use, distribution, and reproduction in any medium, provided the original work is properly cited. 


\section{Z. Lin, S. Chen and T. Gao}

beating to generate directional motions by breaking symmetry (Lauga \& Powers 2009). Additionally, extensive research has been conducted to reveal rich dynamics and new propulsion mechanisms that use the complex fluids' intrinsic anisotropy arising from the non-Newtonian physical and rheological properties. For example, while the extra particle stresses from shear-thinning viscoelastic (e.g. Oldroyd-B) fluids generally impose additional hindrance effects, it has been found that undulatory swimmers, such as $C$. elegans, may leverage both the finite body-length and the polymeric stress relaxation to achieve a higher swimming speed than that in Newtonian fluids (Teran, Fauci \& Shelley 2010; Thomases \& Guy 2014; Salazar, Roma \& Ceniceros 2016). Furthermore, there has been a growing interest in exploring non-equilibrium physics of biosynthetic active materials 'powered' by many-body interactions in complex fluids that are capable of exploiting collective dynamics for useful mechanical work (Ramaswamy 2010; Shelley 2016). Of particular interest is the study of active microparticles (e.g. motile bacteria) in lyotropic liquid crystals (LCs) wherein the extra stress generation is determined by the LC's orientational order, which leads to intriguing swimming behaviours of microswimmers, as well as far-from-equilibrium physical and topological properties of the LCs (Zhou et al. 2014; Lavrentovich 2016; Lintuvuori, Würger \& Stratford 2017; Daddi-Moussa-Ider \& Menzel 2018; Mandal \& Mazza 2019).

So far, there have been several models proposed to study the dynamics of microswimmers in lyotropic LCs. Zhou et al. (2017) employed a $Q$-tensor model (here $Q$-tensor denotes the second-rank orientational order-parameter tensor) derived from the generalized Ericksen-Leslie equation (Sonnet, Maffettone \& Virga 2004) to solve for the orientation field of LCs when a rigid, rodlike particle (B. subtilis) moves in a homeotropic nematic cell where the director is perpendicular to the cell wall. They illustrated how the induced shear determines the ordering patterns (or birefringent cloud) around the moving particle. Using an Ericksen-Leslie model described by the nematic director field (Larson 1999) and enforcing the local director orientation to be tangential to the undulatory body (i.e. tangential anchoring), Krieger, Dias \& Powers (2015a); Krieger, Spagnolie \& Powers (2015b) studied the motion of Taylor's swimming sheet (Taylor 1951), an infinite-length, zero-thickness sheet with imposed small-amplitude travelling waves, in unconfined LCs. The asymptotic solutions they derived suggest that both forward and backward motions can be achieved in different parameter regimes, and the swimmer's mean speed can be either faster or slower than that in a Newtonian fluid. Similar bi-directional swimming motions were reported by the same group when studying the motions of Taylor's swimming sheet in LCs confined by two parallel plates under the tangential anchoring condition (Krieger, Spagnolie \& Powers 2019). In addition to asymptotic analysis, they performed nonlinear simulations using the immersed boundary (IB) method to resolve the fluid-structure interactions of an infinite-length sheet with prescribed finite-amplitude travelling waves. Nevertheless, as pointed out by Krieger et al. (2019), the Ericksen-Leslie model cannot be robustly extended to more general cases where the anchoring direction is misaligned with the director (e.g. homeotropic anchoring), and may break down owing to generations of singularities in the director field.

Motivated by the prior studies and to further seek quantitative understanding of swimming mechanisms in anisotropic fluids, we present a computational framework for studying the undulatory motion of a finite-length microswimmer in a solution of liquid crystal polymers (LCPs), a class of rigid, rodlike aromatic polymers that have much larger sizes and higher aspect ratios than small LC molecules (e.g. para-azoxyanisole). Compared with flexible polymers or molecule aggregates (e.g. lyotropic chromonic LCs Zhou et al. 2014; Zhou 2018), LCPs can much more easily re-orient themselves when 


\section{Q-tensor model for undulatory swimming in a liquid crystal}

interacting with external fields (e.g. fluid flows, electric fields) to show large birefringence (Doi \& Edwards 1988; Larson 1999). As discussed below, the evolution of their orientation distributions can be described by a $Q$-tensor model that is coarse-grained from Doi's kinetic model (Doi 1981; Doi \& Edwards 1988), a prevalent theoretical model for simulating the flow and rheology of LCPs. We treat the microswimmer as a finite-length elastic fibre whose undulatory motion is activated by imposing travelling waves on the body curvature. An IB method is employed to resolve the fluid-structure interactions for the swimmer. The nonlinear simulations of finite-amplitude swimming motions reveal both enhanced and retarded swimming motions in the nematic regime, which correspond to the scenarios when the swimming direction is parallel and perpendicular to the nematic alignment directions, respectively. Our numerical results are qualitatively confirmed by the asymptotic solutions of Taylor's swimming sheet model, and can be further understood by revealing the characteristic near-body flow fields and polymer force distributions.

The paper is organized as follows. Section 2 is dedicated to the mathematical formulation of the problem. We introduce the governing equations for a minimal $Q$-tensor model, and construct an IB formulation. In $\S 3$, we systematically explore the parameter space to study and analyse the finite-amplitude undulatory swimming motions via nonlinear simulations and analytical asymptotic analysis. Finally, we summarize and draw conclusions in $\S 4$. The details of the IB numerical scheme and the derivation steps of the asymptotic solutions of Taylor's swimming sheet are provided in Appendices A and B, respectively.

\section{Mathematical model}

Doi's kinetic model (Doi \& Edwards 1988) adopts a probability distribution function (p.d.f.) $\Psi(\boldsymbol{x}, \boldsymbol{p}, t)$ to describe the ensemble dynamic of concentrated microrods in terms of their centre-of-mass position $\boldsymbol{x}$ and orientation $\boldsymbol{p}(|\boldsymbol{p}|=1)$. When simulating the macro scale hydrodynamic interactions between rods and ambient flows, it is often preferred to use coarse-grained partial differential equations that are derived via proper moment closure owing to their low computation costs by removing $\boldsymbol{p}$ dependency. Consider rodlike polymers with fore-aft symmetry and uniform length $\ell$. In the fluid domain $\Omega_{f}$, a two-dimensional (2-D) model can be derived for the second-moment tensor $\boldsymbol{D}(\boldsymbol{x}, t)=$ $\int_{p} p p \Psi d p$ (Doi 1981; Doi \& Edwards 1988; Feng \& Leal 1997) as

$$
\stackrel{\nabla}{D}+2 E: S=4 \zeta d_{r}(D \cdot D-D: S)-4 d_{r}\left(D-\frac{I}{2}\right)+d_{t} \Delta D,
$$

where $\boldsymbol{D}^{\nabla}=\partial \boldsymbol{D} / \partial t+\boldsymbol{u} \cdot \boldsymbol{\nabla} \boldsymbol{D}-\left(\boldsymbol{D} \cdot \boldsymbol{\nabla} \boldsymbol{u}+\boldsymbol{\nabla} \boldsymbol{u}^{\mathrm{T}} \cdot \boldsymbol{D}\right)$ is the so-called upper-convected time derivative, $\boldsymbol{E}=\left(\boldsymbol{\nabla} \boldsymbol{u}+\nabla \boldsymbol{u}^{\mathrm{T}}\right) / 2$ is the symmetric strain-rate tensor and $\boldsymbol{S}(\boldsymbol{x}, t)=$ $\int_{p} p p p p \Psi d p$ is the fourth-moment tensor. The first term on the right-hand side represents the steric alignment effects arising from the Maier-Saupe potential written as

$$
U_{M S}(\boldsymbol{x}, \boldsymbol{p}, t)=-\zeta p \boldsymbol{p}: \boldsymbol{D}(\boldsymbol{x}, t),
$$

with $\zeta$ as the dimensionless strength coefficient (Maier \& Saupe 1958). The diffusivity coefficients $d_{t}$ and $d_{r}$ originate from the translational and rotational Brownian diffusion motion, respectively. The above equation is a ' $Q$-tensor' model because the trace-free (normalized) order-parameter tensor, the so-called $Q$-tensor, is defined by $Q(x, t)=$ $c^{-1} \boldsymbol{D}-\boldsymbol{I} / 2$, with $c(\boldsymbol{x}, t)=1$ as the concentration field (i.e. zeroth-moment of $\Psi$ ) that remains as a homogeneous constant. In two dimensions, the tensor $Q$ has a maximal 


\section{Z. Lin, S. Chen and T. Gao}

non-negative eigenvalue $\rho$ satisfying $0 \leq \rho \leq 1 / 2$. Assuming that $\rho$ is isolated, then we call its associated unit eigenvector the director $\boldsymbol{m}$, and $0 \leq S=2 \rho \leq 1$ the orientational order parameter. The incompressible fluid velocity $\boldsymbol{u}$ is incorporated in a mean-field fashion, and is governed by the forced Stokes equation:

$$
\begin{gathered}
\nabla \cdot \boldsymbol{u}=0, \\
\nabla p-\mu_{f} \Delta \boldsymbol{u}=\nabla \cdot \boldsymbol{\sigma}_{p}+\boldsymbol{f}_{e},
\end{gathered}
$$

where $\mu_{f}$ is the fluid viscosity and $f_{e}(\boldsymbol{x}, t)$ represents the force exerted by the elastic swimmer. The extra stress of the polymer solution is defined as (Doi 1981; Feng \& Leal 1997)

$$
\sigma_{p}(x, t)=2 v k_{B} T\left(D-\frac{I}{2}\right)-2 v k_{B} T \zeta(D \cdot D-S: D)+\frac{v k_{B} T \beta_{0}}{2 d_{r}\left(v \ell^{3}\right)^{2}} E: S,
$$

where $v \sim \ell^{-3}$ is an effective volume fraction, $k_{B} T$ is the thermal energy scale, $\beta_{0}\left(\nu \ell^{3}\right)^{-2}$ represents a crowdedness factor with $\beta_{0}$ being an empirical coefficient. In this study, we will choose a small empirical crowdedness factor $\beta_{0}\left(\nu \ell^{3}\right)^{-2} \sim 10^{-3}-10^{-4}$ (Feng \& Leal 1997; Feng, Chaubal \& Leal 1998).

Here we have several comments on the above $Q$-tensor model. First, we consider the model to be minimal because (2.2) only describes the nematic elasticity arising from the rods' orientation distribution but ignores spatial heterogeneity. According to Greco \& Marrucci (1992), the Maier-Saupe potential can be further improved by taking the distortion elasticity into account by adding a term proportional to $p \boldsymbol{p}: \Delta \boldsymbol{D}$. However, here we consider the scenarios in the nematic regime where the microswimmer undulations only weakly disturb the LCPs, and the concentration field remains uniform across the domain. For simplicity, we adopt Doi's original model with the Maier-Saupe potential in (2.2). This is consistent with the nonlinear simulation results shown below where the polymer distribution remains spatially homogeneous, with small near-body fluctuations in the orientational order.

Second, as pointed out by Feng, Sgalari \& Leal (2000), adding distortion elasticity into the above $Q$-tensor model can mathematically recover the director formulation of the Ericksen-Leslie model in the limit of weak flow and mild spatial distortion, which can be described by the so-called Frank elasticity with equal (Frank) constants (DeGennes \& Prost 1993). Nevertheless, the director formulation is more suitable for modelling small-molecule LCs that have short orientation relaxation time and hence admit a uniaxial form for the quasi-equilibrium orientational distributions. In comparison, the LCPs' orientations are easily distorted into a non-uniaxial configuration, especially when imposing various flow conditions (e.g. shear and extensional flows). Therefore, the generalized LC theories, either of Ericksen-Leslie or Doi type, which adopt a tensorial order parameter are preferred in simulating LCPs (Beris \& Edwards 1994; Qian \& Sheng 1998; Feng et al. 2000; Sonnet et al. 2004; Klein et al. 2007).

Third, our $Q$-tensor model is essentially non-polar, which has a two-fold meaning. At the micro level, the Doi-Onsager kinetic model adopts Jeffrey's equation to describe the polymers' rotational motion driven by the mean-field fluid velocity gradient and the local alignment torque arising from the Maier-Saupe potential. The resultant rotational flux term hence takes the following form:

$$
\dot{p}=(I-p p) \cdot \nabla \boldsymbol{u} \cdot \boldsymbol{p}-\nabla_{p} U_{M S}(\boldsymbol{x}, \boldsymbol{p})-d_{r} \nabla_{p} \ln \Psi,
$$


where $\nabla_{p}=(\boldsymbol{I}-\boldsymbol{p} \boldsymbol{p}) \cdot \partial / \partial \boldsymbol{p}$ denotes the gradient operator on the unit sphere of orientations. One can prove there is no net-torque exerting on the unit volume of the fluid phase by taking the configurational average of the torque produced by a single test polymer, i.e. $\int_{p} \nabla_{p} U_{M S} \Psi \mathrm{d} p=0$ (Feng et al. 2000). At the macro level, the symmetric extra stress in (2.5) guarantees the conservation of angular momentum in the fluid phase. Moreover, when interior or exterior boundaries are introduced, only the boundary conditions (e.g. no-slip condition) for the velocity field need to be implemented. Solving the coarse-grained evolution equation (2.1) does not require imposing any boundary conditions on $D$ in the limit $d_{t} \rightarrow 0$. Instead, the near-boundary LCPs' orientational distribution is determined by the interplay among the resultant fluid shear, mean-field alignment torque and rotational diffusion as reflected by the right-hand side of (2.6) microscopically.

In the Lagrangian frame $\left(\Omega_{L}\right)$ for the undulatory swimmer of length $L_{S}$, its kinematics can be described by the parametric form $X(s, t)$, with $s$ the local arc length $s \in\left[0, L_{s}\right]$. The actual shape $X(s, t)$ is determined by penalizing deviations from actuation imposed on the body curvature $\kappa_{0}(s, t)$ as

$$
\kappa_{0}(s, t)=-k^{2} A_{0} \sin (k s-\omega t),
$$

starting from an initial configuration given by $X(s, t=0)=\left(s, A_{0}(\sin (k s))\right)$. Equation (2.7) describes the rightward-propagating travelling waves with amplitude $A_{0}$, wavenumber $k$ and angular frequency $\omega$. Imposing actuation in (2.7) produces elastic forces $F_{e}(X)$ along the body, and effectively drive periodic shape changes (or swimming gaits). Following Peskin (2002), the Lagrangian body force can be derived by performing the variational derivative upon the elastic energy $E$, i.e.

$$
\boldsymbol{F}_{e}(X, t)=-\frac{\delta E[X(s, t)]}{\delta X}
$$

The discretized forms for the two components of $\boldsymbol{F}_{e}$ are given in (A2). Here the total elastic energy $E[X]$ include the contributions from both stretching (denoted by subscript $s$ ) and bending (denoted by subscript $b$ ) deformation (Fauci \& Peskin 1988):

$$
E[X(s, t)]=\frac{\sigma_{s}^{0}}{2} \int_{\Omega_{L}}\left(\left|\frac{\partial X}{\partial s}\right|-1\right)^{2} \mathrm{~d} s+\frac{\sigma_{b}^{0}}{2} \int_{\Omega_{L}}\left(\frac{\partial^{2} \boldsymbol{X}}{\partial s^{2}} \cdot \boldsymbol{n}-\kappa_{0}\right)^{2} \mathrm{~d} s
$$

where $\boldsymbol{n}$ denotes the local normal direction. Using the IB method, the Eulerian forcing term $f_{e}$ can be calculated by

$$
f_{e}(x, t)=\int_{\Omega_{L}} F_{e}(s, t) \delta(\boldsymbol{x}-X(s, t)) \mathrm{d} s
$$

where $\delta$ denotes the Dirac delta function that maps between the Eulerian $\left(\Omega_{f}\right)$ and Lagrangian $\left(\Omega_{L}\right)$ domain. Likewise, the Lagrangian velocity can be interpolated from the nearby Eulerian grids via

$$
\frac{\partial X}{\partial t}=\boldsymbol{U}(s, t)=\int_{\Omega_{E}} \boldsymbol{u}(\boldsymbol{x}, t) \delta(X(s, t)-\boldsymbol{x}) \mathrm{d} \boldsymbol{x} .
$$




\section{Z. Lin, S. Chen and T. Gao}

Here we perform mapping between the Eulerian $(x(x, y))$ and the Lagrangian $(X(X, Y))$ domain via a four-point Dirac delta function that is numerically constructed as

$$
\delta(x-X)=\frac{1}{h^{2}} \phi\left(\frac{x-X}{h}\right) \phi\left(\frac{y-Y}{h}\right),
$$

where $h$ is the (uniform) Eulerian mesh width, and the function $\phi(r)$ is constructed as

$$
\phi(r)= \begin{cases}0, & |r| \geq 2, \\ \frac{1}{8}\left(5-2|r|-\sqrt{-7+12|r|-4 r^{2}}\right), & 2 \geq|r| \geq 1, \\ \frac{1}{8}\left(3-2|r|+\sqrt{1+4|r|-4 r^{2}}\right), & 1 \geq|r| \geq 0 .\end{cases}
$$

This choice of the numerical Delta function guarantees the momentum conservation in both the fluid and the solid phases (Peskin 2002).

For non-dimensionalization, we choose the wavelength $\lambda=2 \pi k^{-1}$ as the length scale, wave speed $\omega / k$ as the velocity scale and $2 \nu k_{B} T$ as the LCP's stress scale, which lead to the dimensionless equations in the fluid phase after proper re-scaling:

$$
\begin{gathered}
\nabla \cdot u=0, \\
\nabla p-\Delta u=E r \nabla \cdot \tau_{p}+f_{e}, \\
\tau_{p}=\left(D-\frac{I}{2}\right)-\zeta(D \cdot D-S: D)+\beta E: S, \\
\stackrel{\nabla}{D}+2 E: S=\frac{\zeta}{P e}(D \cdot D-D: S)-\frac{1}{P e}\left(D-\frac{I}{2}\right)+\frac{1}{P e_{t}} \Delta D .
\end{gathered}
$$

Here we define two Péclet numbers, $P e=\omega / 8 \pi d_{r}$ and $P e_{t}=2 \pi \omega / d_{t} k^{2}$, which represent the ratio of the time scales for the rod's rotation and transport over that of undulation (i.e. $\left.\omega^{-1}\right)$, respectively. Especially $P e$ characterizes the time evolution of the orientation field. In this study, we vary $P e \sim O\left(10^{-1}\right)-O(10)$ over three orders of magnitude to fully resolve the non-Newtonian swimming behaviours that are prominent at a finite $P e \sim O(1)$. Meanwhile, $P e_{t} \sim O(10)-O(100)$ is chosen to be at least one order of magnitude higher than $P e$ so that the translational diffusion effect is small or negligible. The coupling between the LCPs and the viscous fluid is characterized by the Ericksen number $E r=$ $4 \pi \nu k_{B} T / \mu_{f} \omega \sim O\left(10^{-1}\right)-O(10)$ (Larson 1999; Krieger et al. 2015b). This parameter choice reveals the strong (weak) flow modification on the polymers' configuration at higher (lower) $E r$ values. When $E r$ becomes even higher (e.g. $E r=20$ ), we notice that the numerical solutions may be difficult to converge. Another dimensionless number in the fluid phase is an effective crowdedness coefficient $\beta=\beta_{0} \omega /\left(8 \pi d_{r}\left(\nu \ell^{3}\right)^{2}\right) \sim O\left(10^{-2}\right)-$ $O\left(10^{-3}\right)$. Equations (2.7)-(2.9) and the two mapping functions (2.10) and (2.11) retain the same mathematical forms, with the two dimensionless stiffness coefficients $\sigma_{s}=$ $\sigma_{s}^{0} 2 \pi k / \omega^{2}, \quad \sigma_{b}=\sigma_{b}^{0} k^{3} / 2 \pi \omega^{2}$. We fix the wavenumber $k=2 \pi$, the swimmer length $L=L_{s} k / 2 \pi=1$ (same as the wavelength) and the amplitude $A=A_{0} k / 2 \pi=0.05$. We choose a large stretching stiffness $\sigma_{s}=O\left(10^{3}\right)$ to enforce inextensibility and vary the bending stiffness $\sigma_{b}=O\left(10^{-3}\right)-O\left(10^{-1}\right)$ to achieve various different swimming gaits. We then use a pseudo-spectral method to solve the above coupled equations in a periodic computation domain. The reader is referred to Appendix A for more details of the numerical algorithm and parameter choice. 
(a)

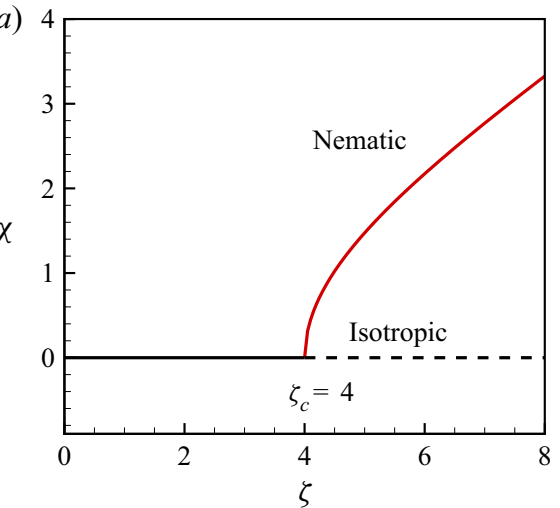

(b)

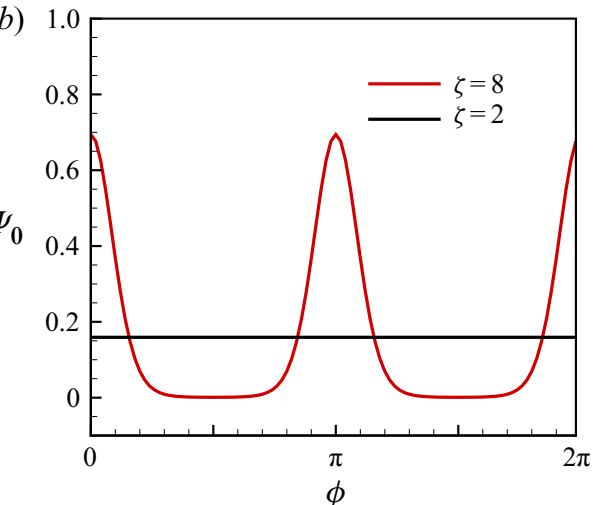

Figure 1. Equilibrium solution $\Psi_{0}$ exhibits a bifurcation across the isotropic-nematic phase transition: (a) $\chi$ as a function of $\zeta$, with the bifurcation occurring at $\zeta_{c}=4 ;(b)$ symmetric distribution of $\Psi_{0}(\phi)$ at $\zeta=2$ and 8 .

\section{Results and discussion}

Without the swimmer, the polymer distribution can be described by the equilibrium solution of the original Doi's kinetic model,

$$
\Psi_{0}=\frac{\exp [\chi \cos (2 \phi)]}{\int_{0}^{2 \pi} \mathrm{d} \phi^{\prime} \exp \left[\chi \cos \left(2 \phi^{\prime}\right)\right]},
$$

where the coefficient $\chi$ satisfies

$$
\chi=\frac{\zeta}{4} \frac{\int_{0}^{2 \pi} \mathrm{d} \phi^{\prime} \cos \left(2 \phi^{\prime}\right) \exp \left[\chi \cos \left(2 \phi^{\prime}\right)\right]}{\int_{0}^{2 \pi} \mathrm{d} \phi^{\prime} \exp \left[\chi \cos \left(2 \phi^{\prime}\right)\right]} .
$$

As shown in figure $1(a), \chi(\zeta)$ admits a bifurcation at $\zeta_{c}=4$, and has two solution branches for the isotropic and nematic states. In figure $1(b)$, the symmetric form of $\Psi_{0}(\phi)$ suggests that the rod's primary (or principal) orientation direction is aligned with the $x$-axis when $\phi=0$ and $\pi$ are in the nematic regime $\left(\zeta \geq \zeta_{c}\right)$, compared with the constant solution in the isotropic regime $\left(0 \leq \zeta<\zeta_{c}\right)$. At each time step in the simulation, we reconstruct $\Psi_{0}(x, p, t)$ of form (3.1) locally in the principal coordinates of the $\boldsymbol{D}(\boldsymbol{x}, t)$ field, and then approximate the fourth moment as

$$
S(x, t) \approx \int_{p} p p p p \Psi_{0}(x, p, t) \mathrm{d} p,
$$

where we follow a quasi-equilibrium ansatz that $D$ and $S$ co-align in principal directions in the eigenspace (see more details in Chaubal \& Leal 1998). This method is also referred to as the Bingham closure (Bingham 1974; Chaubal \& Leal 1998; Feng et al. 1998), which has proven to be accurate in p.d.f. reconstruction and has been widely used in simulating LCPs (Feng et al. 1998; Gao \& Li 2017; Gao et al. 2017).

In the following, we numerically investigate the undulatory motions of a finite-length swimmer in LCPs whose equilibrium configurations are described by $\Psi_{0}$, and focus on the scenarios when the swimmer moves either parallel with or perpendicular to the alignment direction (i.e. $x$-direction) for both the 'stiff' $\left(\sigma_{b}=O\left(10^{-1}\right)\right)$ and 'soft' $\left(\sigma_{b}=O\left(10^{-3}\right)\right)$ cases. In figure $2(a)$, we show the time sequence of the swimmer's shape-change during 
(a)
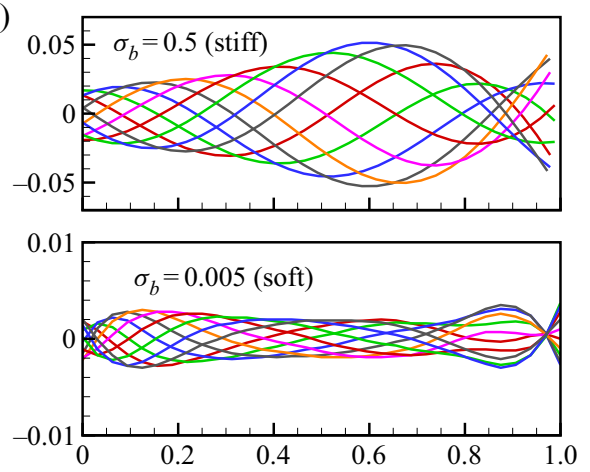

(b)

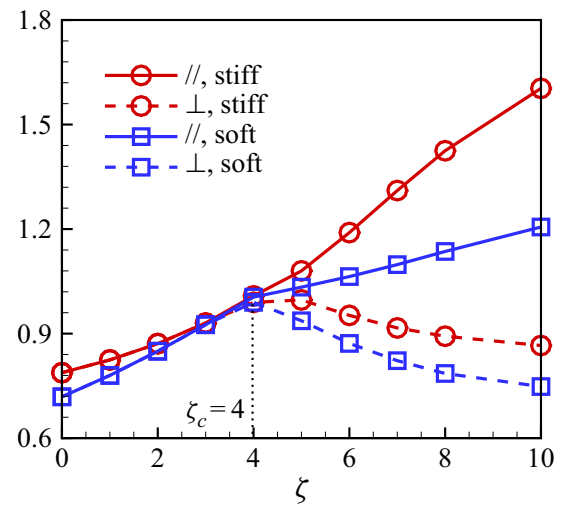

Figure 2. Both stiff and soft undulatory microswimmers exhibit anisotropic swimming behaviours in lyotropic LCPs when measuring the mean centre-of-mass speed $U_{L C}:(a)$ time sequence of swimmer shapes for the stiff (top, $\sigma_{b}=0.5$ ) and the soft (bottom, $\sigma_{b}=0.005$ ) cases during one swimming period; (b) speed ratio $U_{L C} / U_{N}$ as a function of $\zeta$ during the parallel (denoted by ' $/ /$ ') and perpendicular (denoted by ' $\perp$ ') motions when choosing $P e=1$ and $E r=10$.

one swimming period when choosing $\sigma_{b}=0.5$ (top) and $\sigma_{b}=0.005$ (bottom). The stiff swimmer exhibits a wavy pattern of an approximately sinusoidal form with an adequate amplitude $A \approx 0.05$; while the soft swimmer can only slightly bend the body and create much smaller deformations $(A<0.01)$. When imposing rightward-travelling waves on the curvature, leftward unidirectional undulatory motions are observed in all simulations. Hence, we denote the mean centre-of-mass velocity as $-U_{L C} \hat{\boldsymbol{e}}_{x}$ with $\hat{\boldsymbol{e}}_{x}$ a unit basis vector pointing in the positive direction of the $x$-axis. We measure the velocity magnitude $U_{L C}$ by performing time averaging over five undulation periods after the swimming speed reaches quasi-steady states, and then compare it with $U_{N}$ measured in Newtonian fluids. As shown in figure $2(b)$, the speed ratio $U_{L C} / U_{N}$ as a function of $\zeta$ clearly shows non-Newtonian behaviours, especially in the nematic regime where similar bifurcations occur, which correspond the motions that are parallel (denoted by symbol '//') and perpendicular (denoted by symbol ' $\perp$ ') to the $x$-axis. More specifically, we observe enhanced swimming speeds when the swimmer moves parallel in the nematic regime; while retarded motions are seen in the isotropic regime, and in the most nematic regime during perpendicular motions. Additionally, while qualitatively similar behaviours are consistently observed for the stiff and soft cases in the parameter space, it is seen that the stiff swimmer can generally achieve faster mean speeds, which exhibit more significant speed gain and loss. Here it is worthwhile to mention that when the swimming direction is tilted, i.e. with an arbitrary angle between the nematic alignment direction, we observe that (not reported here) the swimmer can perform an entire-body rotation before reaching a steady free swimming. This observation is consistent with the asymptotic solutions of Taylor's swimming sheet in a nematic LC derived by Shi \& Powers (2017). It can be explained by the fact that the anisotropic elastic stress will produce a net torque on the plate when there is any misalignment between the nematic director and the swimming direction.

Figure 3 shows the variations of $U_{L C} / U_{N}$ with respect to $P e$ at various values of $E r$ in both isotropic and nematic regimes. Apparently, for all the cases the speed ratio exhibits stronger non-Newtonian behaviours $\left(U_{L C} / U_{N} \neq 1\right)$ at larger values of $E r$ when fixing $P e$. For the stiff swimmer shown in panels $(a-c)$, at a given $E r, U_{L C} / U_{N}$ is seen to be close to 1 in the small $P e$ regime, which corresponds to a slow undulation (or equivalently, quick rotational diffusion of LCPs). This is because when $P e \rightarrow 0$, the 


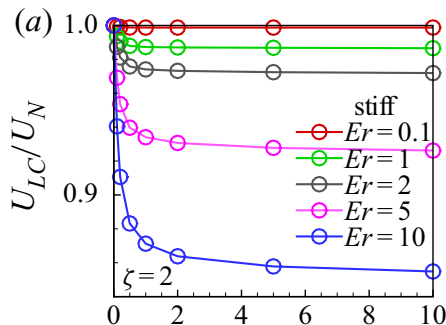

(b)
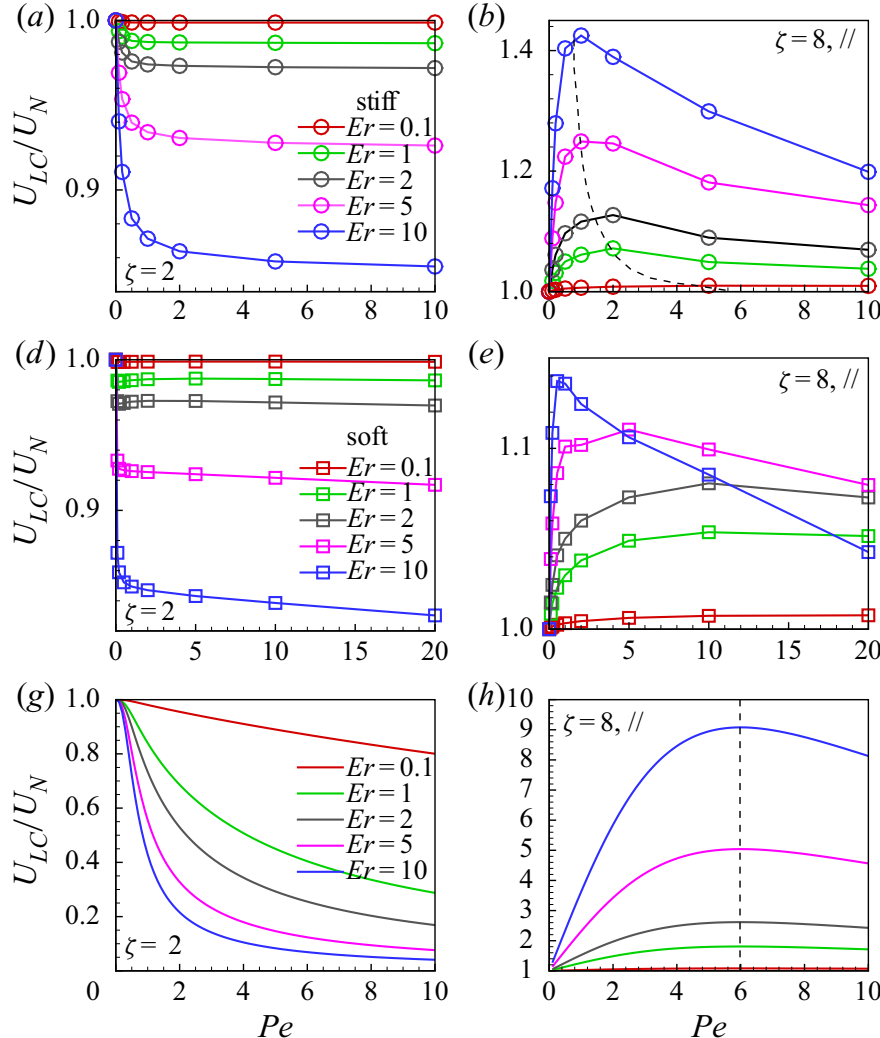

(e)

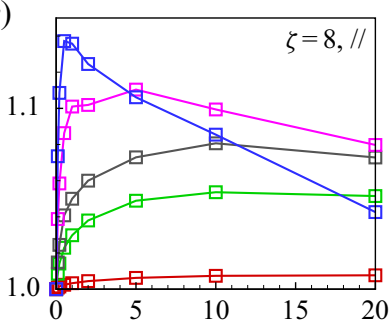

$(h) 10$

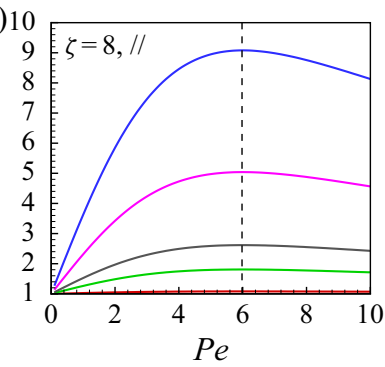

(c)

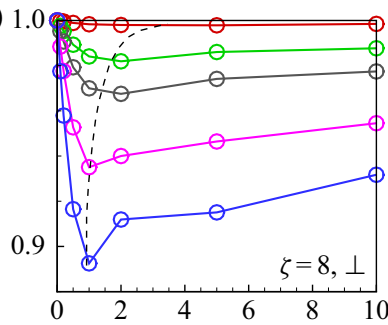

$(f) 1$

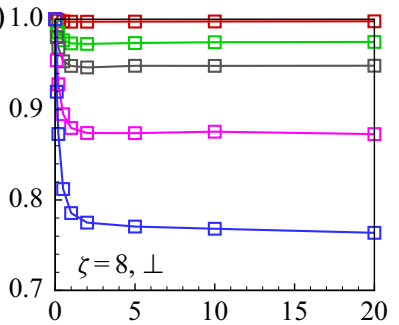

(i)

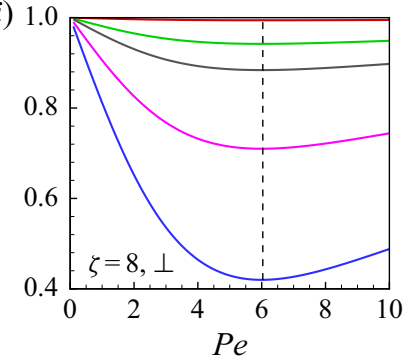

Figure 3. Speed ratio $U_{L C} / U_{N}$ as a function of $P e$ at various values of $E r$ obtained from numerical simulations for the stiff $(a-c)$ and soft $(d-f)$ cases, as well as from the asymptotic solutions for Taylor's swimming sheet $(g-i)$. The black dashed lines in panels $(b, c, h, i)$ mark the locations corresponding to the maximal or minimal values of $U_{L C} / U_{N}$.

rotational diffusion dominates over convection, which not only drives the nematic structure towards equilibrium states (i.e. the right-hand side of (2.17) approximately to be 0 ) but also produces smaller and smaller $\boldsymbol{\tau}_{p}$. At a high $P e$, where convection dominates during fast actuation, the polymer configuration is modulated by flow via constraining stress, i.e. $E: S$, owing to the rod's rigidity, which leads to more complicated behaviours. For a given $E r$, in the isotropic regime, $U_{L C} / U_{N}$ monotonically decreases with respect to $P e$. Nevertheless, in the nematic regime, $U_{L C} / U_{N}$ varies non-monotonically, with the maximum (minimum) occurring at small $P e(\sim O(1))$ during the parallel (perpendicular) swimming motions. Furthermore, in panels $(b, c)$, we mark the extrema locations on the $U_{L C} / U_{N}-P e$ curves using black dashed lines. It is seen that for both parallel and perpendicular cases, the critical values of $P e$ that correspond to the maximal and minimal speed ratio become smaller and smaller when increasing $E r$. In panels $(d-f)$, we show the corresponding results for the soft swimmer cases, and similar trends as for the stiff cases are observed. However, the extrema locations in the nematic regime would be at much larger values of $\mathrm{Pe}$ where the soft swimmer motions become very slow, and hence are not studied here.

To understand the numerical observations, we build an idealized linear model for Taylor's swimming sheet that has an infinite length (Taylor 1951). With the swimmer 


\section{Z. Lin, S. Chen and T. Gao}

moving at the speed $-U_{L C} \hat{\boldsymbol{e}}_{x}$, its vertical displacement can be described as

$$
y(x, t)=\varepsilon \sin (x-t), \quad \varepsilon \ll 1 .
$$

Then we simplify the above governing equations by employing a stream function $\varphi$ via

$$
\boldsymbol{u}=\nabla \times\left(\varphi \hat{\boldsymbol{e}}_{z}\right)
$$

with $\hat{\boldsymbol{e}}_{z}$ as the unit basis vector pointing in the out-of-plane direction, which ensures the incompressibility condition $\boldsymbol{\nabla} \cdot \boldsymbol{u}=0$. By following the solution procedure of Lauga (2007) and Krieger et al. (2015b, 2019), we impose the no-slip condition on the wavy sheet, and perform asymptotic analyses by expanding all the variables in terms of $\varepsilon$ in both the isotropic and nematic regimes. Moreover, we neglect the crowdedness effect (i.e. $\beta=0$ ) in $\tau_{p}$ and the translational diffusion (i.e. $P e_{t}^{-1} \rightarrow 0$ ), and adopt different closure methods for $S$ in the isotropic and nematic regimes to facilitate derivation. After some manipulations (see more derivation details in the supplemental material), we find directional motions only occur in the second order, i.e. $U_{L C}=U_{L C}^{(2)} \varepsilon^{2}$, and the speed ratio can be calculated as

$$
\begin{aligned}
& \frac{U_{L C}}{U_{N}}=1-\frac{16 E r P e^{3}}{(4+E r P e)\left[(4-\zeta)^{2}+16 P e^{2}\right]}, \quad \text { (Isotropic) } \\
& \frac{U_{L C}}{U_{N}}=1+\frac{4 \operatorname{ErPe}(\zeta-2)}{(\zeta-2)^{2}+P e^{2}}\left(D_{11}^{(0)}-\frac{1}{2}\right) D_{11}^{(0)}, \quad \text { (Nematic) }
\end{aligned}
$$

where $D_{11}^{(0)}=\left(\zeta \pm \sqrt{\zeta^{2}-2 \zeta}\right) / 2 \zeta$ (also see (B41)) is the diagonal component of the equilibrium solution $D^{(0)}=\operatorname{diag}\left(D_{11}^{(0)}, 1-D_{11}^{(0)}\right)$, and the plus (minus) sign corresponds to parallel (perpendicular) swimming motion with respect to the alignment direction. Unlike the possible bi-directional motions predicted by Krieger et al. (2015b), it is straightforward to show that $U_{L C} / U_{N}>0$ for the cases of isotropic (3.6) and parallel swimming in the nematic regime (3.7); while for the perpendicular swimming cases, (3.7) suggests that for a given $P e, U_{L C} / U_{N}<0$ only occurs at very large $E r$ values, which are far above the practical parameter range. Hence the asymptotic model predicts universal unidirectional motions that are in the opposite direction of the prescribed travelling wave, which are also consistent with our numerical results of finite-length swimmers. Moreover, as shown in figure $3(g-i)$, the asymptotic solutions predict the qualitatively similar behaviours of $U_{L C} / U_{N}$ as the numerical results in figure 3(a-f). However, (3.7) suggests the maximal and minimal speed ratios all occur at $P e=\zeta-2$ for both the parallel and perpendicular cases and are independent of $E r$. This analytical prediction is different from the numerical results that depend on both $P e$ and $E r$, which is likely owing to the finite-length effect of the swimmer (see more discussion below).

In the isotropic regime, the monotonic decay as a function of $P e$ at a given $E r$ is reminiscent of the behaviours in viscoelastic (e.g. Oldroyd-B) fluids (Lauga 2007; Shen \& Arratia 2011). This can be witnessed by introducing an effective polymer viscosity $\mu_{p}$ (Doi \& Edwards 1988; Feng \& Leal 1997) as $\mu_{p} / \mu_{f}=\alpha(S) E r P e$, where ErPe defines the effective polymer contribution to the zero-shear-rate viscosity, and the estimated coefficient $\alpha$ characterizes the dependence on the order parameter $S$. When $\zeta \rightarrow 0$, 


\section{Q-tensor model for undulatory swimming in a liquid crystal}

(a)

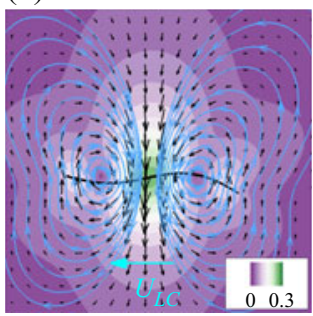

(e)

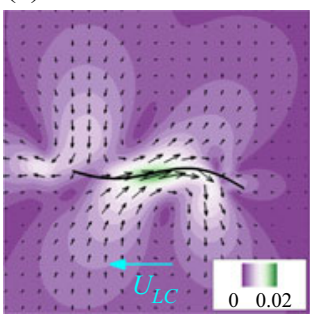

(b)

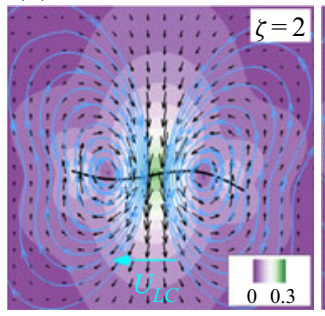

$(f)$

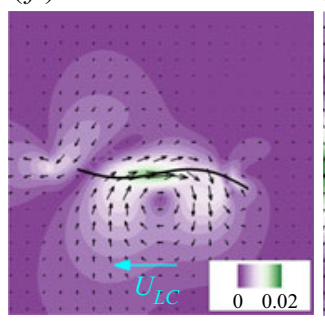

(c)

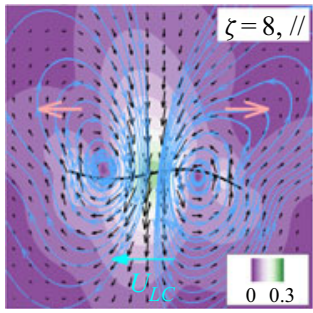

$(g)$

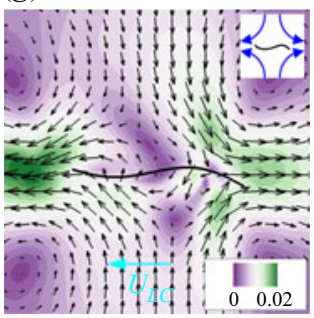

$(d)$

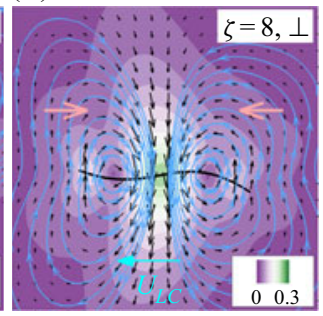

(h)

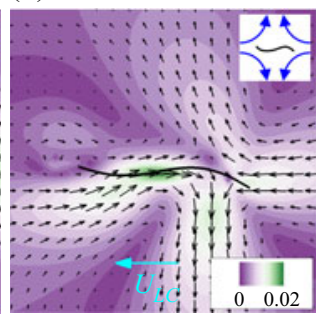

Figure 4. The instantaneous $(a-d)$ and time-averaged $(e-h)$ velocity field around a 'stiff' swimmer in the body-fixed frame when choosing $\sigma_{b}=0.5, P e=1$ and $E r=10$ : $(a, e)$ Newtonian; $(b, f)$ isotropic, $\zeta=2 ;(c, g)$ nematic and parallel, $\zeta=8 ;(d, h)$ nematic and perpendicular, $\zeta=8$. The vector field and the background colour correspond to the velocity $\boldsymbol{u}$ and its magnitude $|\boldsymbol{u}|$.

we estimate $S=0$ and $\alpha(S)=1$, and hence can rewrite (3.6) as

$$
\frac{U_{L C}}{U_{N}}=\frac{1+\left(\frac{4 \mu_{f}}{4 \mu_{f}+\mu_{p}}\right) P e^{2}}{1+P e^{2}}, \quad(\zeta \rightarrow 0)
$$

which resembles the asymptotic solution for Taylor's swimming sheet in viscoelastic fluids (Lauga 2007). In the nematic regime, from (3.7), apparently the speed gain and loss are seen to be directly determined by the horizontal $\left(D_{11}^{(0)}>1 / 2\right)$ and vertical $\left(D_{11}^{(0)}<1 / 2\right)$ alignment directions. Moreover, $U_{L C} / U_{N} \rightarrow 1$ is observed in the limit of $P e \rightarrow \infty$, which suggests diminishing non-Newtonian behaviours for very fast undulations in sharply aligned LCPs. The reader is referred to Appendix B for more details of the derivation.

Next, we take a closer look at the flow patterns to uncover the nonlinear effect arising from the finite length of the swimmer. As shown in figure 4(a-d), we compare the simulation results of the instantaneous flow fields at the end of each period, after the swimming motions reach quasi-steady states. While the isotropic case in panel $(b)$ resembles the Newtonian one in panel $(a)$, the parallel (perpendicular) swimming case in the nematic regime in panel $(c)$ (panel $d$ ) has slightly wider (narrower) circulation zones near the two ends. Indeed, when averaging over three to five periods, we consistently find the flow patterns shown in figure $4(e-h)$ have distinctive features. Compared with the Newtonian case in panel $(e)$, the isotropic flow in panel $(f)$ appears to be slightly weakened. More interestingly, the mean flow field in the nematic regime appears to be extensile (panel $g$ ) and contractile (panel $h$ ), which correspond to the parallel and perpendicular motions, respectively.

Figure $5(a-c)$ reveal the characteristics of the nematic field. Without imposing particular anchoring conditions for $\boldsymbol{D}$ along the wavy body, we find the time-averaged (denoted by ' \langle\rangle ') director distribution $\langle\boldsymbol{m}\rangle$ approximately follows the isotropic (panel $a$ ) and aligned 
(a)

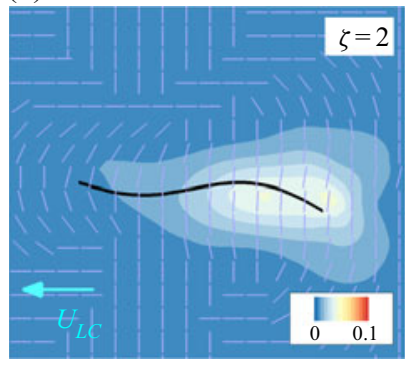

(d)

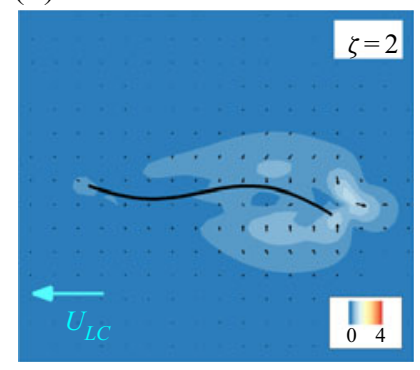

(b)

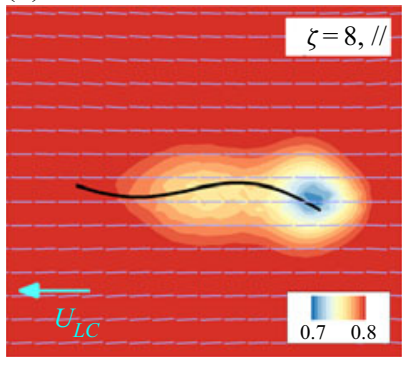

(e)

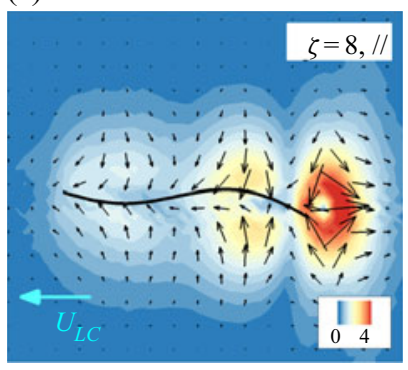

(c)

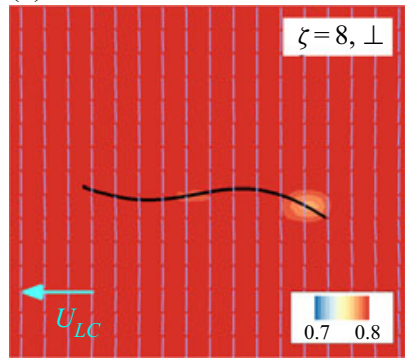

$(f)$

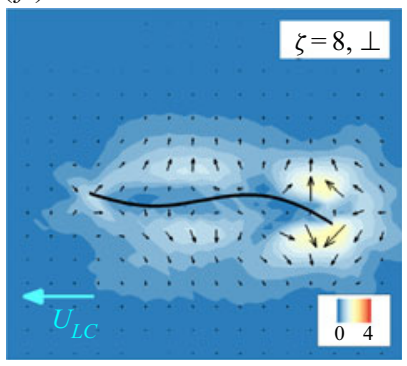

Figure 5. The time-averaged director and polymer force fields around a stiff swimmer corresponding to figure $4(f-h):(a-c)$ nematic director $\langle\boldsymbol{m}\rangle$ superposed on the colourmap of the order parameter $S ;(d-f)$ polymer force vector $\left\langle f_{p}\right\rangle$ superimposed on the force magnitude $\left|\left\langle f_{p}\right\rangle\right|$.

(panels $b, c$ ) configurations. However, owing to the finite length of the swimmer, the resultant structures lack left-right symmetry. The disturbances on the nematic field are concentrated in the areas around the body's rear side without propagating further. High-orientational orders suggests enforcement of local alignment owing to strong steric interactions characterized by large values of $\zeta$. When comparing the two scenarios in panels $(b)$ and $(c)$, it appears that parallel motions can impact larger areas. This is because the vertical oscillations during undulation are a lot faster $(\sim 5-10$ times) than the resultant horizontal migration, and can lead to stronger momentum exchange along the $y$-direction. Hence, it is easier for the shear-induced force to re-orient the LCPs perpendicularly, which leaves large low-order zones during parallel motions.

Further examination of the time-averaged polymer force, $\left\langle f_{p}\right\rangle=\left\langle\nabla \cdot \tau_{p}\right\rangle$ in figure 5 , reveals more precise propulsion mechanisms. Because the periodic elastic body force has a zero mean, i.e. $\left\langle f_{e}\right\rangle=0$ from (2.15), the near-body fluid flows are driven by $\left\langle f_{p}\right\rangle$ at a finite $E r$. As typically shown in panel $(d)$, isotropic LCPs only permit small $\left\langle f_{p}\right\rangle$ without showing clear correlations with the swimming direction. In comparison, nematic LCPs lead to much stronger, asymmetrical $\left\langle f_{p}\right\rangle$ in panel $(e, f)$, with the largest values near the 'tail.' Additionally, they are clearly seen to serve as the driving forces of the extensile and contractile flow patterns in figures $4(g)$ and $4(h)$, respectively. More intriguingly, unlike the isotropic cases, the near-body distributions of $\left\langle f_{p}\right\rangle$ in the nematic regime highly correlate with the swimming direction. In figure $5(e)$, the projected force vectors at $y=0$ all approximately point leftwards to 'push' the swimmer forward; while in panel $(f)$, the projected force along the $x$-direction, while weaker, appears to be overall pointing rightward to 'pull' the swimmer backward. 


\section{Q-tensor model for undulatory swimming in a liquid crystal}

\section{Conclusion}

This work has built a $Q$-tensor model to study the nonlinear dynamics of undulatory swimming motions in lyotropic LCPs in the limit of vanishing Reynolds numbers. Combining direct numerical simulations and asymptotic analysis, we have revealed both enhanced and retarded swimming behaviours for a flexible swimmer moving in rigid, rodlike polymers. Compared with those top-down macro models of Ericksen-Leslie or Landau-de Gennes (DeGennes \& Prost 1993) for small-molecule LCs, ours is derived bottom-up, and has more accurate microscopic origins and fewer computation parameters. Hence it provides a simple and convenient computation framework to model fluid-structure interactions in lyotropic LCs. Our results suggest that in addition to adopting wavy body undulations to break symmetry, it is possible for a finite-length flexible swimmer to make use of the unique near-body fluid dynamics and polymer force generation to adjust the migration speed. We expect to apply the same methodology to generally study a microorganism's motion, both individually and collectively, in anisotropic complex-fluid environments.

Acknowledgements. Z.L. acknowledges the Fundamental Research Funds for the Central Universities of China grant no. 2020QNA4046. T.G. acknowledges the National Science Foundation grant no. 1943759. The anonymous reviewers' are thanked for their constructive comments that helped improve and clarify this manuscript.

Declaration of interests. The authors report no conflict of interest.

Author ORCIDs.

(D) Tong Gao https://orcid.org/0000-0001-5049-8538.

\section{Appendix A. Immersed boundary solver}

In the flexible fibre, the elastic body force $F_{e}\left(F_{x}, F_{y}\right)$ is calculated explicitly at each time step. Following the IB approaches (Fauci \& Peskin 1988; Lee \& Wolgemuth 2016), we discretize the variational derivative of the bending energy functional, and derive the following component form for the $i$ th node at the position $X_{i}\left(X_{i}, Y_{i}\right)$ :

$$
\begin{aligned}
F_{x, i}= & \frac{\sigma_{s}}{\Delta s}\left[\left(\frac{\left|X_{i+1}-X_{i}\right|}{\Delta s}-1\right)\left(\frac{X_{i+1}-X_{i}}{\left|X_{i+1}-X_{i}\right|}\right)-\left(\frac{\left|X_{i}-X_{i-1}\right|}{\Delta s}-1\right)\left(\frac{X_{i}-X_{i-1}}{\left|X_{i}-X_{i-1}\right|}\right)\right] \\
& -\frac{\sigma_{b}}{\Delta s^{3}}\left(\left(\kappa-\kappa_{0}\right)_{i-1}\left(Y_{i-1}-Y_{i-2}\right)-\left(\kappa-\kappa_{0}\right)_{i}\left(Y_{i+1}-Y_{i-1}\right)\right. \\
& \left.+\left(\kappa-\kappa_{0}\right)_{i+1}\left(Y_{i+2}-Y_{i+1}\right)\right), \\
F_{y, i}= & \frac{\sigma_{s}}{\Delta s}\left[\left(\frac{\left|X_{i+1}-X_{i}\right|}{\Delta s}-1\right)\left(\frac{Y_{i+1}-Y_{i}}{\left|X_{i+1}-X_{i}\right|}\right)-\left(\frac{\left|X_{i}-X_{i-1}\right|}{\Delta s}-1\right)\left(\frac{Y_{i}-Y_{i-1}}{\left|X_{i}-X_{i-1}\right|}\right)\right] \\
& +\frac{\sigma_{b}}{\Delta s^{3}}\left(\left(\kappa-\kappa_{0}\right)_{i-1}\left(X_{i-1}-X_{i-2}\right)-\left(\kappa-\kappa_{0}\right)_{i}\left(X_{i+1}-X_{i-1}\right)\right. \\
& \left.+\left(\kappa-\kappa_{0}\right)_{i+1}\left(X_{i+2}-X_{i+1}\right)\right),
\end{aligned}
$$

where the curvature is defined as $\kappa_{i}=\hat{\boldsymbol{e}}_{z} \cdot\left(\boldsymbol{X}_{i+1}-\boldsymbol{X}_{i}\right) \times\left(\boldsymbol{X}_{i}-\boldsymbol{X}_{i-1}\right) / \Delta s^{3}$ and $\Delta s=$ $L / N_{s}$.

In the fluid phase, we first solve the constitutive equations for the velocity $\boldsymbol{u}$ and the second-moment tensor $\boldsymbol{D}$. Here we use the method of Vaithianathan \& Collins (2003) to enforce the symmetric positive definiteness of $\boldsymbol{D}$ whose diagonal components are bounded, 
i.e. $\operatorname{tr}(\boldsymbol{D})=D_{11}+D_{22}=1$ and $0 \leq D_{11}, D_{22} \leq 1$. We apply the Cholesky decomposition on $D$ to obtain

$$
\boldsymbol{D}=\boldsymbol{L} \cdot \boldsymbol{L}^{\mathrm{T}}=\left(\begin{array}{ll}
l_{11} & 0 \\
l_{21} & l_{22}
\end{array}\right)\left(\begin{array}{cc}
l_{11} & l_{21} \\
0 & l_{22}
\end{array}\right)
$$

where $L$ is a lower triangular matrix. Then we perform another transformation for the components of $L$,

$$
l_{11}=\frac{1}{\pi} \arctan \eta+\frac{1}{2}, \quad l_{22}=\frac{1}{\pi} \arctan \gamma+\frac{1}{2}, \quad l_{21}=\frac{2}{\pi} \arctan \chi,
$$

so that the parameters $(\eta, \gamma, \chi)$ are now unconstrained. Substituting ((A3) and (A4a-c)) into the evolution equation of $\boldsymbol{D}$ leads to

$$
\left.\begin{array}{c}
\frac{\partial \eta}{\partial t}+u_{x} \frac{\partial \eta}{\partial x}+u_{y} \frac{\partial \eta}{\partial y}=\pi\left(1+\eta^{2}\right)\left[\begin{array}{c}
l_{11} \frac{\partial u_{x}}{\partial x}+l_{21} \frac{\partial u_{x}}{\partial y}-\frac{1}{2 P e}\left(l_{11}-\frac{1}{2 l_{11}}\right) \\
+\frac{1}{P e_{t}} \frac{1}{2 l_{11}} \Delta D_{11}-\frac{1}{2 l_{11}} K_{11}
\end{array}\right] \\
\frac{\partial \chi}{\partial t}+u_{x} \frac{\partial \chi}{\partial x}+u_{y} \frac{\partial \chi}{\partial y}=\frac{\pi}{2}\left(1+\chi^{2}\right)\left[\begin{array}{c}
l_{11} \frac{\partial u_{y}}{\partial x}+\frac{l_{22}^{2}}{l_{11}} \frac{\partial u_{x}}{\partial y}-l_{21} \frac{\partial u_{x}}{\partial x}-\frac{1}{2 P e}\left(l_{21}+\frac{l_{21}}{2 l_{11}^{2}}\right) \\
-\frac{1}{P e_{t}} \frac{l_{21}}{2 l_{11}^{2}} \Delta D_{11}+\frac{1}{P e_{t}} \frac{1}{l_{11}} \Delta D_{12}+\frac{l_{21}}{2 l_{11}^{2}} K_{11}-\frac{1}{l_{11}} K_{12}
\end{array}\right] \\
\frac{\partial \gamma}{\partial t}+u_{x} \frac{\partial \gamma}{\partial x}+u_{y} \frac{\partial \gamma}{\partial y}=\pi\left(1+\gamma^{2}\right)\left[\begin{array}{c}
l_{22} \frac{\partial u_{y}}{\partial y}-\frac{l_{22} l_{21}}{l_{11}} \frac{\partial u_{x}}{\partial y}+\frac{1}{2 P e}\left(\frac{l_{21}^{2}}{2 l_{22} l_{11}^{2}}-l_{22}+\frac{1}{2 l_{22}}\right) \\
+\frac{1}{P e_{t}} \frac{l_{21}^{2}}{2 l_{22} l_{11}^{2}} \Delta D_{11}-\frac{1}{P e_{t}} \frac{l_{21}}{l_{22} l_{11}} \Delta D_{12}+\frac{1}{P e_{t}} \frac{1}{2 l_{22}} \Delta D_{22} \\
-\frac{l_{21}^{2}}{2 l_{22} l_{11}^{2}} K_{11}+\frac{l_{21}}{l_{22} l_{11}} K_{12}-\frac{1}{2 l_{22}} K_{22}
\end{array}\right] \cdot
\end{array}\right\}
$$

In the above a shorthand notation $K=2 E: S-(\zeta / P e)(D \cdot D-D: S)$ is introduced and computed explicitly.

When $D$ is obtained, we evaluate the polymer stress $\tau_{p}$, and then directly compute the 2-D velocity field $\boldsymbol{u}=\left(u_{x}, u_{y}\right)$ in the Fourier space as

$$
\tilde{\boldsymbol{u}}=\frac{1}{\xi^{2}}(I-\hat{\xi} \hat{\xi}) \cdot \tilde{f}_{\text {total }}
$$

where $f_{\text {total }}=\operatorname{Er} \boldsymbol{\nabla} \cdot \boldsymbol{\tau}_{p}+\boldsymbol{f}_{e}, \hat{\boldsymbol{\xi}}$ is the wavevector with $|\boldsymbol{\xi}|=\xi(\hat{\boldsymbol{\xi}}=\boldsymbol{\xi} / \xi)$. The equations in the fluid phase are solved using a pseudo-spectral method, with the time integration being evaluated using the fourth-order Runge-Kutta method. For all simulations performed in this study, we choose the computation parameters as $\Omega_{f}=2 \times 2, h=1 / 128$, $\Delta t=10^{-5}-10^{-4}, P e=0.1-10, P e_{t}=10-100, E r=1-10, \zeta=0-10, \beta=0.0005-0.005$, $N_{s}=32, L=1, A=0.05, k=2 \pi, \omega=2 \pi, \sigma_{b}=0.005-0.5, \sigma_{s}=3000 \sigma_{b}$. As shown in figure $6(a-c)$ for the time-dependent velocity components, we verified the numerical results by performing convergence tests for a parallel moving swimmer.

Moreover, as shown in figure 7, we performed another benchmark study for the undulatory swimming motions in an Oldroyd-B (OB) fluid where the dimensionless Deborah $(D e)$ number, which plays a similar role as $P e$ in the LCP cases, is defined as the wave frequency by the OB fluid relaxation time. We measured the mean centre-of-mass swimming speed $U_{O B}$ of the swimmer that has a long body length $L=4$, and compared 

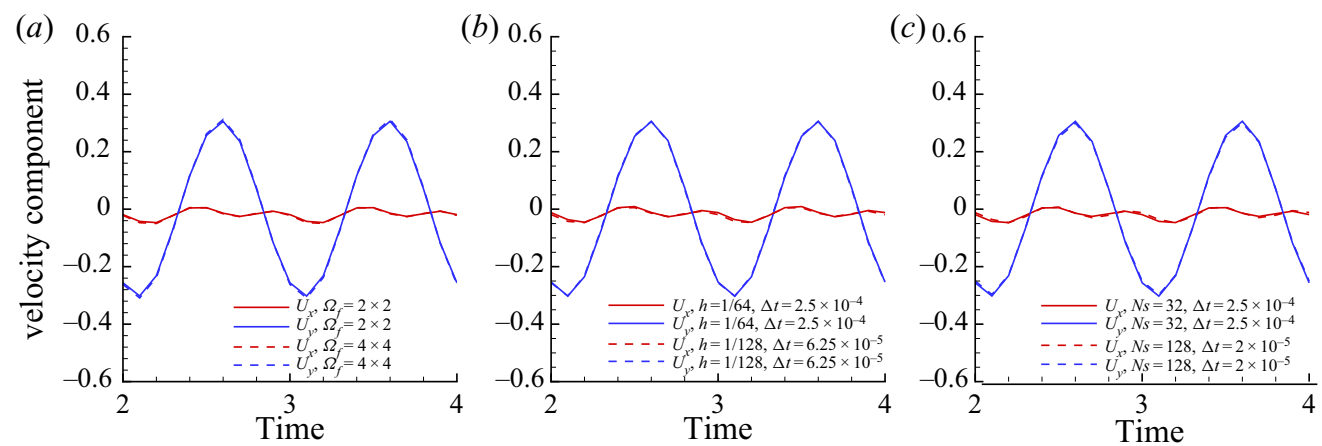

Figure 6. Convergence tests of a parallel moving swimmer with the time-dependent centre-of-mass velocity $U\left(U_{x}, U_{y}\right)$ by varying: $(a)$ domain size $\left(\Delta t=6.25 \times 10^{-5}, h=1 / 128\right)$; $(b)$ Eulerian grid width $\left(\Omega_{f}=2 \times 2\right)$; and $(c)$ Lagrangian grid spacing $\left(\Omega_{f}=2 \times 2, h=1 / 128\right)$. These parameters are fixed: $P e=10 ; P e_{t}=100$; $E r=1 ; \beta=0.005 ; \zeta=8 ; L=1 ; A=0.05 ; k=2 \pi ; \omega=2 \pi ; \sigma_{b}=0.5 ; \sigma_{s}=1500$.

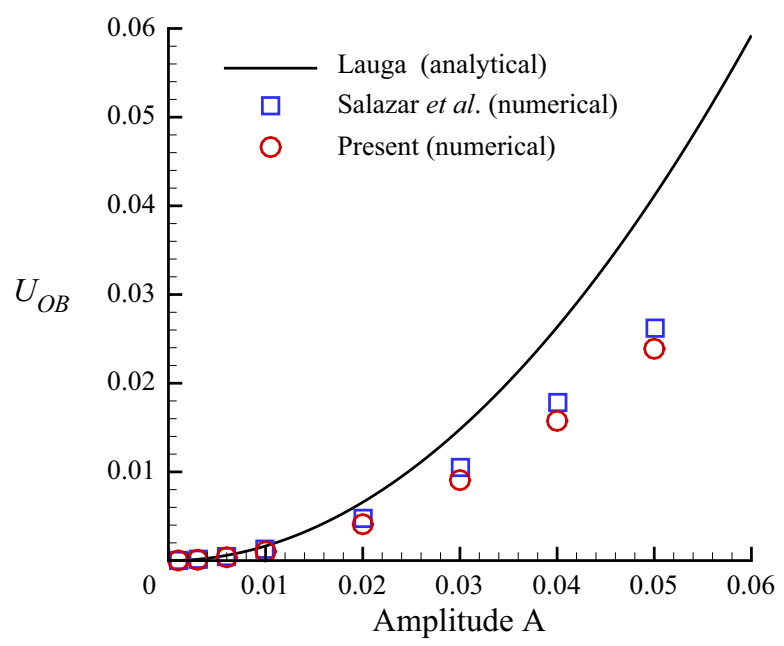

Figure 7. Time-averaged centre-of-mass speed $U_{O B}$ for undulatory swimming motion in an Oldroyd-B fluid. These parameters are fixed: $\Omega_{f}=8 \times 8 ; h=1 / 64 ; N_{s}=64 ; \eta_{p} / \eta_{s}=1 / 2 ; L=4, D e=1 ; \Delta t=2.5 \times 10^{-4}$.

the speed ratio with the numerical data of Salazar et al. (2016) and the asymptotic results for Taylor's swimming sheet of Lauga (2007),

$$
\frac{U_{O B}}{U_{N}}=\frac{1+\left(\frac{\eta_{s}}{\eta_{s}+\eta_{p}}\right), D e^{2}}{1+D e^{2}},
$$

where $\eta_{s}$ and $\eta_{p}$ respectively represent the solvent and polymer contribution to the viscosity. The Newtonian speed $U_{N}$ can be derived as

$$
U_{N}=\frac{1}{2}\left(\frac{\omega}{k}\right)(A k)^{2}+O(A k)^{4},
$$

where $k=2 \pi$ is the wavenumber. We find our results agree well with the previous studies.

In the third test, we examined the impact of varying bending stiffness when choosing high values of $\sigma_{b}$. As shown in figure 8 for the time-dependent velocity, only small 


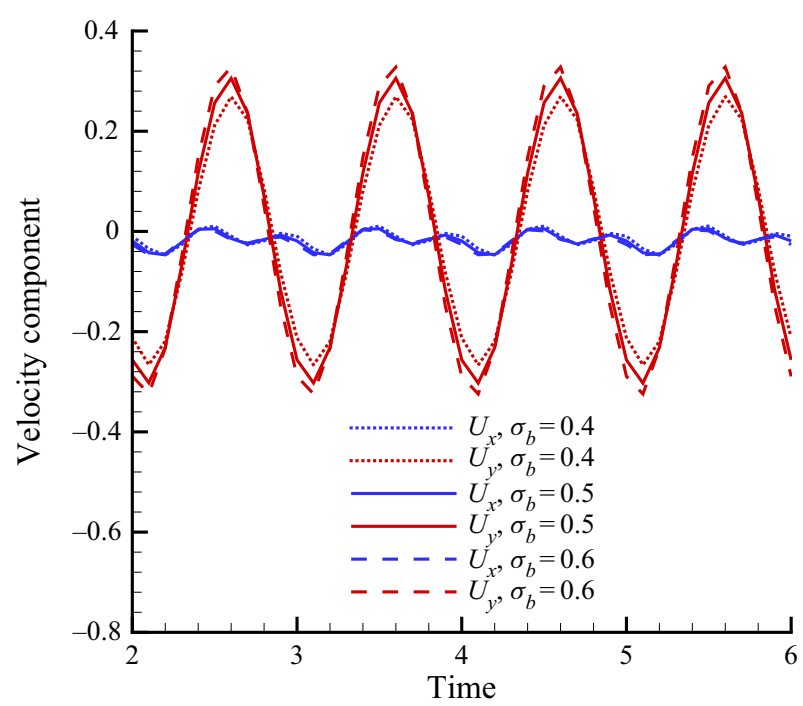

Figure 8 . Time-dependent centre-of-mass velocity $U\left(U_{x}, U_{y}\right)$ for a parallel moving swimmer when choosing different values of bending stiffness $\sigma_{b}$. These parameters are fixed: $\Omega_{f}=2 \times 2 ; \Delta t=6.25 \times 10^{-4} ; h=$ $1 / 128 ; P e=10 ; P e_{t}=100 ; E r=1 ; \beta=0.005 ; \zeta=8 ; L=1 ; N_{s}=32 ; A=0.05 ; k=2 \pi ; \omega=2 \pi ; \sigma_{s}=$ 1500 .

differences are seen when $\sigma_{b}$ goes beyond 0.5 . In these scenarios, the swimmer can quickly respond to the imposed target curvature and well follow the travelling-wave actuation. Hence, in the main text, we choose $\sigma_{b}=0.5$ for the cases of a stiff swimmer.

\section{Appendix B. Asymptotic solution of Taylor's swimming sheet}

In the moving frame of the swimmer, we consider the vertical displacement of an infinite-length wavy sheet with the described travelling-wave motion $y(x, t)=A \sin (k x-$ $\omega t$ ). When choosing $1 / k$ as the length scale, $1 / \omega$ as the time scale and $\omega / k$ as the velocity scale, the dimensionless form can be written as $y(x, t)=\varepsilon \sin (x-t)$. Here we assume a small amplitude $\varepsilon=A k \ll 1$. Following Lauga (2007), we adopt a streamfunction $\varphi(x, y, t)$ such that the 2-D velocity components can be computed as

$$
u_{x}=\frac{\partial \varphi}{\partial y}, \quad u_{y}=-\frac{\partial \varphi}{\partial x}
$$

with the incompressibility condition being satisfied. The boundary conditions for $\varphi(x, y, t)$ arise from conditions at infinity and on the undulatory sheet with a steady speed $-U_{L C} \hat{\boldsymbol{e}}_{x}$. Then the far-field condition at $y=\infty$ reads

$$
\left.\nabla \varphi\right|_{(x, \infty)}=U_{L C} \hat{\boldsymbol{e}}_{y} .
$$

On the swimming sheet, the no-slip velocity condition is imposed as

$$
\left.\nabla \varphi\right|_{(x, \varepsilon \sin (x-t))}=\varepsilon \cos (x-t) \hat{\boldsymbol{e}}_{x} .
$$

Recalling the forced Stokes equation,

$$
\nabla p=\Delta \boldsymbol{u}+\operatorname{Er} \nabla \cdot \boldsymbol{\tau}_{p},
$$


$Q$-tensor model for undulatory swimming in a liquid crystal

the polymer stress, when neglecting $\beta$, is written as

$$
\tau_{p}=\left(D-\frac{I}{2}\right)-\zeta(D \cdot D-D: S)
$$

By ignoring the translational diffusion, the $D$ evolution equation is written as

$$
\stackrel{\nabla}{D}+2 E: S=-\frac{1}{P e}\left(D-\frac{I}{2}\right)+\frac{\zeta}{P e}(D \cdot D-D: S) .
$$

When applying the curl on both sides of (B4), we have

$$
\nabla \times\left(\nabla \cdot \tau_{p}\right)=\frac{1}{E r} \nabla^{4} \varphi
$$

Next, we expand all the variables in terms of $\varepsilon$ up to the second order as

$$
\begin{gathered}
\varphi=\varepsilon \varphi^{(1)}+\varepsilon^{2} \varphi^{(2)}+O\left(\varepsilon^{3}\right), \\
\boldsymbol{\tau}=\boldsymbol{\tau}^{(0)}+\varepsilon \boldsymbol{\tau}^{(1)}+\varepsilon^{2} \boldsymbol{\tau}^{(2)}+O\left(\varepsilon^{3}\right), \\
D=D^{(0)}+\varepsilon \boldsymbol{D}^{(1)}+\varepsilon^{2} D^{(2)}+O\left(\varepsilon^{3}\right), \\
U_{L C}=\varepsilon U_{L C}^{(1)}+\varepsilon^{2} U_{L C}^{(2)}+O\left(\varepsilon^{3}\right) .
\end{gathered}
$$

After some manipulations, we can derive the following governing equations. zeroth-order:

$$
\begin{gathered}
\frac{\partial \boldsymbol{D}^{(0)}}{\partial t}+\boldsymbol{u}^{(0)} \cdot \nabla \boldsymbol{D}^{(0)}-\left(\boldsymbol{D}^{(0)} \cdot \nabla \boldsymbol{u}^{(0)}+\nabla \boldsymbol{u}^{(0)^{\mathrm{T}}} \cdot \boldsymbol{D}^{(0)}\right)+2 \boldsymbol{E}^{(0)}: \boldsymbol{S}^{(0)} \\
=-\frac{1}{P e}\left(\boldsymbol{D}^{(0)}-\frac{\boldsymbol{I}}{2}\right)+\frac{\zeta}{P e}\left(\boldsymbol{D}^{(0)} \cdot \boldsymbol{D}^{(0)}-\boldsymbol{D}^{(0)}: \boldsymbol{S}^{(0)}\right) \\
\boldsymbol{\tau}_{p}^{(0)}=\left(\boldsymbol{D}^{(0)}-\frac{\boldsymbol{I}}{2}\right)-\zeta\left(\boldsymbol{D}^{(0)} \cdot \boldsymbol{D}^{(0)}-\boldsymbol{D}^{(0)}: \boldsymbol{S}^{(0)}\right)
\end{gathered}
$$

First-order:

$$
\begin{aligned}
& \frac{\partial \boldsymbol{D}^{(1)}}{\partial t}+\boldsymbol{u}^{(0)} \cdot \nabla \boldsymbol{D}^{(1)}+\boldsymbol{D}^{(1)} \cdot \nabla \boldsymbol{D}^{(0)} \\
& -\left(D^{(0)} \cdot \nabla \boldsymbol{u}^{(1)}+\boldsymbol{D}^{(1)} \cdot \nabla \boldsymbol{u}^{(0)}+\nabla \boldsymbol{u}^{(0)^{\mathrm{T}}} \cdot \boldsymbol{D}^{(1)}+\nabla \boldsymbol{u}^{(1)^{\mathrm{T}}} \cdot \boldsymbol{D}^{(0)}\right) \\
& +2\left(E^{(0)}: S^{(1)}+E^{(1)}: S^{(0)}\right)=-\frac{1}{P e} D^{(1)} \\
& +\frac{\zeta}{P e}\left(D^{(0)} \cdot D^{(1)}+D^{(1)} \cdot D^{(0)}-D^{(0)}: S^{(1)}-D^{(1)}: S^{(0)}\right), \\
& \tau_{p}^{(1)}=D^{(1)}-\zeta\left(D^{(0)} \cdot D^{(1)}+D^{(1)} \cdot D^{(0)}-D^{(0)}: S^{(1)}-D^{(1)}: S^{(0)}\right) .
\end{aligned}
$$




\section{Z. Lin, S. Chen and T. Gao}

Second-order:

$$
\begin{gathered}
\frac{\partial D^{(2)}}{\partial t}+u^{(0)} \cdot \nabla D^{(2)}+u^{(1)} \cdot \nabla D^{(1)}+u^{(2)} \cdot \nabla D^{(0)} \\
-\left(D^{(0)} \cdot \nabla u^{(2)}+D^{(1)} \cdot \nabla u^{(1)}+D^{(2)} \cdot \nabla u^{(0)}+\nabla u^{(0)} \cdot D^{(2)}+\nabla u^{(1)} \cdot D^{(1)}+\nabla u^{(2)}{ }^{\mathrm{T}} \cdot D^{(0)}\right) \\
+2\left(E^{(0)}: S^{(2)}+E^{(1)}: S^{(1)}+E^{(2)}: S^{(0)}\right)=-\frac{1}{P e} D^{(2)} \\
+\frac{\zeta}{P e}\left(D^{(0)} \cdot D^{(2)}+D^{(1)} \cdot D^{(1)}+D^{(2)} \cdot D^{(0)}-D^{(0)}: S^{(2)}-D^{(1)}: S^{(1)}-D^{(2)}: S^{(0)}\right), \\
\tau_{p}^{(2)}=D^{(2)}-\zeta\left(D^{(0)} \cdot D^{(2)}+D^{(1)} \cdot D^{(1)}+D^{(2)} \cdot D^{(0)}-D^{(0)}: S^{(2)}-D^{(1)}: S^{(1)}-D^{(2)}: S^{(0)}\right)
\end{gathered}
$$

Homogeneous solutions are admitted at the zeroth-order. To solve for the first-order solutions, we note that the corresponding boundary conditions become

$$
\begin{gathered}
\left.\nabla \varphi^{(1)}\right|_{(x, \infty)}=U_{L C}^{(1)} \hat{\boldsymbol{e}}_{y}, \\
\left.\nabla \varphi^{(1)}\right|_{(x, 0)}=\cos (x-t) \hat{\boldsymbol{e}}_{x} .
\end{gathered}
$$

In the above, the no-slip boundary condition has been projected from the wavy body onto the $x$-axis, i.e. at $y=0$. Similarly, at the second-order, they can be derived as

$$
\begin{gathered}
\left.\nabla \varphi^{(2)}\right|_{(x, \infty)}=U_{L C}^{(2)} \hat{\boldsymbol{e}}_{y} \\
\left.\nabla \varphi^{(2)}\right|_{(x, 0)}=-\left.\sin (x-t) \nabla\left(\frac{\partial \varphi^{(1)}}{\partial y}\right)\right|_{(x, 0)} .
\end{gathered}
$$

\section{B.1. Isotropic cases}

In the isotropic regime where $0 \leq \zeta<\zeta_{c}$, we close the fourth-moment tensor $S$ by expanding the p.d.f. near the isotropy as

$$
\Psi=\frac{1}{2 \pi}+\frac{2}{\pi}\left(p p-\frac{I}{2}\right): D,
$$

which leads to

$$
\begin{gathered}
S_{i j k l}=-\frac{1}{24}\left(\delta_{i j} \delta_{k l}+\delta_{i k} \delta_{j l}+\delta_{i l} \delta_{j k}\right) \\
+\frac{1}{6}\left(\delta_{i j} D_{k l}+\delta_{i k} D_{j l}+\delta_{i l} D_{j k}+\delta_{j k} D_{i l}+\delta_{j l} D_{i k}+\delta_{k l} D_{i j}\right) .
\end{gathered}
$$

At the zeroth-order, we obtain $\boldsymbol{u}^{(0)}=0, \boldsymbol{\tau}_{p}^{(0)}=0$, and $\boldsymbol{D}^{(0)}=\operatorname{diag}(1 / 2,1 / 2)$. At the first-order, we substitute $\boldsymbol{D}^{(0)}$ into (B15) and obtain

$$
\boldsymbol{\tau}_{p}^{(1)}=\left(1-\frac{\zeta}{4}\right) D^{(1)}
$$




\section{Q-tensor model for undulatory swimming in a liquid crystal}

When substituting (B24) into (B14), we can get

$$
\left(\frac{4}{4-\zeta}\right) \frac{\partial \boldsymbol{\tau}_{p}^{(1)}}{\partial t}=-\frac{1}{P e} \boldsymbol{\tau}_{p}^{(1)}+\frac{1}{2} E^{(1)} .
$$

After taking the divergence and then applying the curl on both sides of (B25), we obtain

$$
\left[\frac{4}{\operatorname{Er}(4-\zeta)} \frac{\partial}{\partial t}+\left(\frac{1}{\operatorname{ErPe}}+\frac{1}{4}\right)\right] \nabla^{4} \varphi^{(1)}=0 .
$$

Given the boundary conditions in ((B18)-(B19)), it is straightforward to solve for the first-order solutions as

$$
\begin{gathered}
\varphi^{(1)}(x, y, t)=(1+y) e^{-y} \sin (x-t), \\
U_{L C}^{(1)}=0 .
\end{gathered}
$$

At the second-order, we first evaluate (B17) to obtain

$$
\boldsymbol{\tau}_{p}^{(2)}=\left(1-\frac{\zeta}{4}\right) D^{(2)}-\zeta\left(D^{(1)} \cdot D^{(1)}-D^{(1)}: S^{(1)}\right) .
$$

Then (B16) can be rewritten as

$$
\begin{gathered}
\frac{4}{4-\zeta} \frac{\partial \tau_{p}^{(2)}}{\partial t}+\frac{1}{P e} \tau_{p}^{(2)}-\frac{1}{2} E^{(2)}=-2 E^{(1)}: S^{(1)}-\boldsymbol{u}^{(1)} \cdot \nabla \boldsymbol{D}^{(1)} \\
-\left(\boldsymbol{D}^{(1)} \cdot \nabla \boldsymbol{u}^{(1)}+\nabla \boldsymbol{u}^{(1)} \cdot \boldsymbol{D}^{(1)}\right)-\frac{4 \zeta}{4-\zeta} \frac{\partial\left(\boldsymbol{D}^{(1)} \cdot \boldsymbol{D}^{(1)}-\boldsymbol{D}^{(1)}: \boldsymbol{S}^{(1)}\right)}{\partial t},
\end{gathered}
$$

which allows us to derive the governing equation for $\varphi^{(2)}$ as

$$
\begin{aligned}
& {\left[\frac{4}{\operatorname{Er}(4-\zeta)} \frac{\partial}{\partial t}+\left(\frac{1}{E r P e}+\frac{1}{4}\right)\right] \nabla^{4} \varphi^{(2)}=\nabla \times\left\{\nabla \cdot \left[-2 E^{(1)}: S^{(1)}-\boldsymbol{u}^{(1)} \cdot \nabla \boldsymbol{D}^{(1)}\right.\right.} \\
& \left.\left.\quad-\left(\boldsymbol{D}^{(1)} \cdot \boldsymbol{\nabla} \boldsymbol{u}^{(1)}+\nabla \boldsymbol{u}^{(1)^{\mathrm{T}}} \cdot \boldsymbol{D}^{(1)}\right)-\frac{4 \zeta}{4-\zeta} \frac{\partial\left(\boldsymbol{D}^{(1)} \cdot \boldsymbol{D}^{(1)}-\boldsymbol{D}^{(1)}: \boldsymbol{S}^{(1)}\right)}{\partial t}\right]\right\}
\end{aligned}
$$

Finally, to solve for mean speed $U_{L C}$, we can perform the time averaging (' \langle\rangle ') as the following:

$$
\left(\frac{1}{E r P e}+\frac{1}{4}\right) \nabla^{4}\left\langle\varphi^{(2)}\right\rangle=\frac{4 P e^{2}}{(4-\zeta)^{2}+16 P e^{2}}\left(8 y^{2}-24 y+8\right) e^{-2 y}
$$

with boundary conditions in ((B20)-(B21)) now becoming

$$
\begin{gathered}
\frac{\partial\left\langle\varphi^{(2)}\right\rangle}{\partial y}(\infty)=U_{L C}^{(2)}, \\
\frac{\partial\left\langle\varphi^{(2)}\right\rangle}{\partial y}(0)=\frac{1}{2} .
\end{gathered}
$$

We can then obtain

$$
\frac{\mathrm{d}}{\mathrm{d} y}\left\langle\varphi^{(2)}\right\rangle=a_{0}\left(\frac{1}{2}-y^{2}\right) e^{-2 y}+\left(\frac{1}{2}-\frac{1}{2} a_{0}\right),
$$


where $a_{0}=(E r P e /(4+E r P e)) 16 P e^{2} /\left((4-\zeta)^{2}+16 P e^{2}\right)$. Eventually we can evaluate the mean swimming speed at the second-order as

$$
\frac{U_{L C}}{U_{N}}=\frac{U_{L C}^{(2)}}{U_{N}^{(2)}}=1-\frac{16 E r P e^{3}}{(4+E r P e)\left[(4-\zeta)^{2}+16 P e^{2}\right]},
$$

where $U_{N}=U_{N}^{(2)} \varepsilon^{2}=\varepsilon^{2} / 2$ is the mean swimming speed in a Newtonian fluid. Note that in Doi's theory, the polymer contribution to the zero-shear-rate viscosity can be effectively defined as (Feng \& Leal 1997)

$$
\frac{\mu_{p}}{\mu_{f}}=\alpha(S) E r P e,
$$

where $S=\sqrt{(D: D-1 / 2)}$ is the order parameter, $\alpha(S)$ is a concentration coefficient and $\mu_{p}$ represents the polymer contribution to the viscosity. Hence, when $\zeta=0$, we estimate $S=0$ and $\alpha(S)=1$, which leads to

$$
\frac{U_{L C}}{U_{N}}=\frac{1+\left(\frac{\mu_{f}}{\mu_{f}+\mu_{p} / 4}\right) P e^{2}}{1+P e^{2}} .
$$

\section{B.2. Nearly-aligned cases}

In the deep nematic regime, we adopt a classical quadratic closure to approximate $S$ (Doi \& Edwards 1988):

$$
S=D D
$$

which corresponds to the strong alignment configuration with large $\zeta$ values when $\zeta>\zeta_{c}$. At the zeroth-order, we solve for the equilibrium solution $D^{(0)}=\operatorname{diag}\left(D_{11}^{(0)}, 1-D_{11}^{(0)}\right)$ via

$$
\zeta\left\{D_{11}^{(0)}-\left[D_{11}^{(0)^{2}}+\left(1-D_{11}^{(0)}\right)^{2}\right] D_{11}^{(0)}\right\}-\left(D_{11}^{(0)}-\frac{1}{2}\right)=0
$$

which admits the solutions

$$
D_{11}^{(0)}=\frac{\zeta \pm \sqrt{\zeta^{2}-2 \zeta}}{2 \zeta}
$$

The plus (minus) sign in the above equation represents the scenario when the nematic alignment direction is along the $x$ - $(y$-) axis. Hence, when fixing the swimmer's moving direction to be horizontal (vertical), the above solution corresponds to the parallel (perpendicular) swimming cases.

At the first-order, it is straightforward to show that

$$
\boldsymbol{\tau}_{p}^{(1)}=(\zeta-2) D_{11}^{(1)}\left(\begin{array}{ll}
1 & 0 \\
0 & -1
\end{array}\right) .
$$

Substituting (B42) into (B14), we have

$$
\left.\begin{array}{c}
\frac{1}{\zeta-2} \frac{\partial \tau_{p, 11}^{(1)}}{\partial t}+4\left(D_{11}^{(0)}-1\right) D_{11}^{(0)} E_{11}^{(1)}+\frac{1}{P e} \tau_{p, 11}^{(1)}=0 \\
\frac{1}{\zeta-2} \frac{\partial \tau_{p, 22}^{(1)}}{\partial t}-4\left(D_{11}^{(0)}-1\right) D_{11}^{(0)} E_{11}^{(1)}+\frac{1}{P e} \tau_{p, 22}^{(1)}=0
\end{array}\right\}
$$




\section{Q-tensor model for undulatory swimming in a liquid crystal}

Similar to the derivation of (B7), we can obtain

$$
\left[\frac{1}{(\zeta-2) E r} \frac{\partial}{\partial t}+\frac{1}{E r P e}\right] \nabla^{4} \varphi^{(1)}+\frac{4}{\zeta} \frac{\partial^{4} \varphi^{(1)}}{\partial x^{2} \partial y^{2}}=0
$$

To facilitate further analytical manipulations, we consider the strong alignment cases when $\zeta$ is large $(\zeta \gg 1)$, which allows us to neglect the last term in (B44), and obtain the same first-order solution as (B27).

At the second-order, using the equilibrium solutions of $\boldsymbol{D}^{(0)}$, we show that

$$
\begin{gathered}
\boldsymbol{\tau}_{p}^{(2)}=\left[(\zeta-2) D_{11}^{(2)}+\zeta\left(3 D_{11}^{(1)^{2}}+D_{12}^{(1)^{2}}\right)\left(2 D_{11}^{(0)}-1\right)\right]\left(\begin{array}{ll}
1 & 0 \\
0 & -1
\end{array}\right) \\
+2 \zeta D_{11}^{(1)} D_{12}^{(1)}\left(2 D_{11}^{(0)}-1\right)\left(\begin{array}{cc}
0 & 1 \\
1 & 0
\end{array}\right),
\end{gathered}
$$

which leads to

$$
\left.\begin{array}{l}
\left(\frac{1}{\zeta-2}\right) \frac{\partial}{\partial t}\left[\tau_{p, 11}^{(2)}-\zeta\left(3 D_{11}^{(1)^{2}}+D_{12}^{(1)^{2}}\right)\left(2 D_{11}^{(0)}-1\right)\right]+G_{11}+\frac{1}{P e} \tau_{p, 11}^{(2)}=0 \\
\left(\frac{1}{\zeta-2}\right) \frac{\partial}{\partial t}\left[\tau_{p, 22}^{(2)}+\zeta\left(3 D_{11}^{(1)^{2}}+D_{12}^{(1)}\right)\left(2 D_{11}^{(0)}-1\right)\right]+G_{22}+\frac{1}{P e} \tau_{p, 22}^{(2)}=0
\end{array}\right\}
$$

where

$$
\begin{gathered}
G=\boldsymbol{u}^{(1)} \cdot \nabla D^{(1)}-\left(D^{(0)} \cdot \nabla u^{(2)}+D^{(1)} \cdot \nabla u^{(1)}+\nabla u^{(1)^{\mathrm{T}}} \cdot D^{(1)}+\nabla \boldsymbol{u}^{(2)^{\mathrm{T}}} \cdot \boldsymbol{D}^{(0)}\right) \\
+2\left(\boldsymbol{E}^{(1)}: \boldsymbol{S}^{(1)}+\boldsymbol{E}^{(2)}: \boldsymbol{S}^{(0)}\right) .
\end{gathered}
$$

In the end, we can derive the linear equation for $\varphi^{(2)}$ as

$$
\begin{gathered}
\left(\frac{1}{\zeta-2}\right) \frac{\partial}{\partial t}\left[\frac{1}{E r} \nabla^{4} \varphi^{(2)}+2 \frac{\partial^{2}\left(\zeta\left(3 D_{11}^{(1)^{2}}+D_{12}^{(1)^{2}}\right)\left(2 D_{11}^{(0)}-1\right)\right)}{\partial x \partial y}\right]+\frac{1}{E r P e} \nabla^{4} \varphi^{(2)} \\
\quad+\frac{\partial^{2}\left(G_{22}-G_{11}\right)}{\partial x \partial y}=\left(\frac{1}{\zeta-2} \frac{\partial}{\partial t}+\frac{1}{P e}\right)\left(\frac{\partial^{2}}{\partial x^{2}}-\frac{\partial^{2}}{\partial y^{2}}\right)\left[2 \zeta D_{11}^{(1)} D_{12}^{(1)}\left(2 D_{11}^{(0)}-1\right)\right]
\end{gathered}
$$

Similar to the isotropic case, here we perform the time averaging of (B48) as

$$
\nabla^{4}\left\langle\varphi^{(2)}\right\rangle=\frac{2 \operatorname{ErPe}(\zeta-2)}{P e^{2}+(\zeta-2)^{2}}\left(2 D_{11}^{(0)}-1\right)\left[4 y^{2}+\left(8 D_{11}^{(0)}-12\right) y-\left(8 D_{11}^{(0)}-6\right)\right] e^{-2 y} .
$$

When applying the boundary conditions ((B20)-(B21)), we obtain

$$
\frac{\mathrm{d}}{\mathrm{d} y}\left\langle\varphi^{(2)}\right\rangle=-\frac{a_{1}}{2}\left(y^{2}+2 D_{11}^{(0)} y+D_{11}^{(0)}\right) e^{-2 y}+\left(\frac{1}{2}+\frac{a_{1} D_{11}^{(0)}}{2}\right),
$$


where $a_{1}=\left(2(\zeta-2) \operatorname{ErPe} /\left((\zeta-2)^{2}+P e^{2}\right)\right)\left(2 D_{11}^{(0)}-1\right)$. So we can eventually solve for the speed ratio at the second-order as

$$
\frac{U_{L C}}{U_{N}}=1+\frac{4 \operatorname{ErPe}(\zeta-2)}{(\zeta-2)^{2}+P e^{2}}\left(D_{11}^{(0)}-\frac{1}{2}\right) D_{11}^{(0)}
$$

\section{REFERENCES}

BERIS, A.N. \& EDWARDS, B.J. 1994 Thermodynamics of flowing systems: with internal microstructure. Oxford Engineering Science Series, Oxford University Press.

Bingham, C. 1974 An antipodally symmetric distribution on the sphere. Ann. Stat. 2, 1201-1225.

Chaubal, C. \& LEAL, L. 1998 A closure approximation for liquid-crystalline polymer models based on parametric density estimation. J. Rheol. 42, 177-201.

Daddi-Moussa-IDer, A. \& Menzel, A.M. 2018 Dynamics of a simple model microswimmer in an anisotropic fluid: implications for alignment behavior and active transport in a nematic liquid crystal. Phys. Rev. Fluids 3, 094102.

DeGennes, P.G. \& Prost, J. 1993 The Physics of Liquid Crystals. Oxford University Press.

DoI, M. 1981 Molecular dynamics and rheological properties of concentrated solutions of rodlike polymers in isotropic and liquid crystalline phases. J. Polym. Sci. Polym. Phys. Ed. 19, 229-243.

DoI, M. \& EDWARDS, S. 1988 The Theory of Polymer Dynamics. Oxford University Press.

FAUCI, L. \& PESkin, C. 1988 A computational model of aquatic animal locomotion. J. Comput. Phys. 77, $85-108$.

Feng, J., Chaubal, C. \& Leal, L. 1998 Closure approximations for the Doi theory: which to use in simulating complex flows of LCPs. J. Rheol. 42, 1095-1119.

FENG, J. \& LEAL, L. 1997 Simulating complex flows of liquid-crystalline polymers using the Doi theory. J. Rheol. 41, 1317-1335.

FENG, J., SGALARI, G. \& LEAL, L. 2000 A theory for flowing nematic polymers with orientational distortion. J. Rheol. 44, 1085-1101.

Gao, T., Betterton, M., Jhang, A. \& Shelley, M. 2017 Analytical structure, dynamics, and coarse-graining of a kinetic model of an active fluid. Phys. Rev. Fluids 2, 093302.

GAO, T. \& LI, Z. 2017 Self-driven droplet powered by active nematics. Phys. Rev. Lett. 119, 108002.

GreCo, F. \& MARRUCCI, G. 1992 Molecular structure of the hedgehog point defect in nematics. Mol. Cryst. Liq. Cryst. 210, 129-141.

Klein, D.H., Leal, L.G., García-Cervera, C.J. \& Ceniceros, H.D. 2007 Ericksen number and deborah number cascade predictions of a model for liquid crystalline polymers for simple shear flow. Phys. Fluids 19, 023101.

Krieger, M., Dias, M. \& Powers, T. 2015a Minimal model for transient swimming in a liquid crystal. Eur. Phys. J. E 38, 94.

Krieger, M., Spagnolie, S. \& Powers, T. 2015b Microscale locomotion in a nematic liquid crystal. Soft Matt. 11, 9115-9125.

Krieger, M., Spagnolie, S. \& Powers, T. 2019 Swimming with small and large amplitude waves in a confined liquid crystal. J. Non-Newtonian Fluid Mech. 273, 104185.

LARSON, R.G. 1999 The Structure and Rheology of Complex Fluids. Oxford University Press.

LaUga, E. 2007 Propulsion in a viscoelastic fluid. Phys. Fluids 19 (8), 083104.

Lauga, E. \& Powers, T. 2009 The hydrodynamics of swimming microorganisms. Rep. Prog. Phys. 72 (9), 096601.

LaVrentovich, O. 2016 Active colloids in liquid crystals. Curr. Opin. Colloid Interface Sci. 21, 97-109.

Lee, P. \& Wolgemuth, C.W. 2016 An immersed boundary method for two-phase fluids and gels and the swimming of caenorhabditis elegans through viscoelastic fluids. Phys. Fluids 28 (1), 011901.

Lintuvuori, J.S., WÜRger, A. \& StratFord, K. 2017 Hydrodynamics defines the stable swimming direction of spherical squirmers in a nematic liquid crystal. Phys. Rev. Lett. 119, 068001.

MAIER, W. \& SAUPE, A. 1958 Eine einfache molekulare theorie des nematischen kristallinflüssigen zustandes. Zeit. Nat. Teil A 13, 564.

MANDAL, S. \& MAZZA, M.G. 2019 Multiparticle collision dynamics for tensorial nematodynamics. Phys. Rev. E 99, 063319.

PESKIN, C.S. 2002 The immersed boundary method. Acta Numer. 10, 479-517.

PurCell, E.M. 1977 Life at low Reynolds number. Am. J. Phys. 45, 3-11. 


\section{Q-tensor model for undulatory swimming in a liquid crystal}

Qian, T. \& Sheng, P. 1998 Generalized hydrodynamic equations for nematic liquid crystals. Phys. Rev. E 58, 7475-7485.

Ramaswamy, S. 2010 The mechanics and statistics of active matter. Annu. Rev. Cond. Matt. Phys. 1, 323-345.

Salazar, D., Roma, A. \& Ceniceros, H. 2016 Numerical study of an inextensible, finite swimmer in Stokesian viscoelastic flow. Phys. Fluids 28, 063101.

SHELlEy, M. 2016 The dynamics of microtubule/motor-protein assemblies in biology and physics. Annu. Rev. Fluid Mech. 48, 487-506.

Shen, X. \& Arratia, P. 2011 Undulatory swimming in viscoelastic fluids. Phys. Rev. Lett. 106, 208101.

SHI, J. \& Powers, T. 2017 Swimming in an anisotropic fluid: how speed depends on alignment angle. Phys. Rev. Fluids 2, 123102.

Sonnet, A., Maffettone, P. \& Virga, E. 2004 Continuum theory for nematic liquid crystals with tensorial order. J. Non-Newtonian Fluid Mech. 119, 51-59.

TAYlor, G.I. 1951 Analysis of the swimming of microscopic organisms. Proc. R. Soc. Lond. A $209,447$.

Teran, J., Fauci, L. \& Shelley, M. 2010 Viscoelastic fluid response can increase the speed and efficiency of a free swimmer. Phys. Rev. Lett. 104, 038101.

Thomases, B. \& GuY, R. 2014 Mechanisms of elastic enhancement and hindrance for finite-length undulatory swimmers in viscoelastic fluids. Phys. Rev. Lett. 113, 098102.

VAithianATHAN, T \& COLlins, L.R. 2003 Numerical approach to simulating turbulent flow of a viscoelastic polymer solution. J. Comput. Phys. 187 (1), 1-21.

ZHOU, S. 2018 Recent progresses in lyotropic chromonic liquid crystal research: elasticity, viscosity, defect structures, and living liquid crystals. Liq. Cryst. Today 27, 91-108.

Zhou, S., Sokolov, A., Lavrentovich, O. \& Aranson, I. 2014 Living liquid crystals. Proc. Natl Acad. Sci. 111, 1265-1270.

Zhou, S., Tovkach, O., Golovaty, D., Sokolov, A., Aranson, I. \& Lavrentovich, O. 2017 Dynamic states of swimming bacteria in a nematic liquid crystal cell with homeotropic alignment. New J. Phys. 19, 055006. 